\title{
Weighted Stepanov-like pseudo-almost automorphic mild solutions for semilinear fractional differential equations
}

Bing He, Junfei Cao* and Bicheng Yang

${ }^{\text {*Correspondence: }}$

jfcaomath@163.com;

caojunfei@gdei.edu.cn

Department of Mathematics,

Guangdong University of

Education, Xingangzhong Road,

Guangzhou, 510310, P.R. China

\begin{abstract}
This work is concerned with the existence and uniqueness of weighted Stepanov-like pseudo-almost automorphic mild solutions for a class of semilinear fractional differential equations, $D_{t}^{\alpha} x(t)=A x(t)+D_{t}^{\alpha-1} F(t, x(t)), t \in \mathbb{R}$, where $1<\alpha<2, A$ is a linear densely defined operator of sectorial type of $\omega<0$ on a complex Banach space $X$ and $F$ is an appropriate function defined on phase space. The fractional derivative is understood in the Riemann-Liouville sense. The results obtained are utilized to study the existence and uniqueness of weighted Stepanov-like pseudo-almost automorphic mild solutions for a fractional relaxation-oscillation equation.
\end{abstract}

Keywords: weighted Stepanov-like pseudo-almost automorphic function; semilinear fractional differential equation; fractional relaxation-oscillation equation; solution operator; fractional integral

\section{Introduction}

In this paper, we are concerned with the existence and uniqueness of weighted Stepanovlike pseudo-almost automorphic mild solutions for the following semilinear fractional differential equations:

$$
D_{t}^{\alpha} x(t)=A x(t)+D_{t}^{\alpha-1} F(t, x(t)), \quad t \in \mathbb{R},
$$

where $1<\alpha<2$,

$$
A: D(A) \subset X \rightarrow X
$$

is a linear densely defined operator of sectorial type of $\omega<0$ on a complex Banach space $X$, and

$$
F: \mathbb{R} \times X \rightarrow X
$$

is an appropriate function. The fractional derivative is understood in the RiemannLiouville sense.

The almost periodic function was introduced seminally by Bochner in 1927 [1]. It plays an important role in describing the phenomena that are similar to the periodic oscillations

\section{Springer}

(c) 2015 He et al.; licensee Springer. This is an Open Access article distributed under the terms of the Creative Commons Attribution License (http://creativecommons.org/licenses/by/4.0), which permits unrestricted use, distribution, and reproduction in any medium, provided the original work is properly credited. 
Table 1 Historical development of almost periodicity

\begin{tabular}{ll}
\hline Function & Original reference \\
\hline Almost periodic (AP) & Bochner [1] \\
Asymptotic almost periodic (AAP) & Fréchet [16] \\
Pseudo almost periodic (PAP) & Zhang [17] \\
Weighted pseudo-almost periodic (WPAP) & Diagana [18] \\
Stepanov-like almost periodic (SP AP) & Stepanov [19, 20] \\
Stepanov-like pseudo-almost periodic (SPAP) & Diagana [21] \\
Weighted Stepanov-like pseudo-almost periodic (SP WPAP) & Diagana et al. [22] \\
\hline
\end{tabular}

which can be observed frequently in many fields, such as celestial mechanics, nonlinear vibration, electromagnetic theory, plasma physics, engineering, ecosphere, and so on [24]. In mathematics, the almost periodic functions are closely connected with harmonic analysis, differential equations, dynamical systems, and so on [5], they are the generalization of continuous periodic and quasi-periodic functions. In the last several decades, the basic theories on the almost periodic functions have been well developed [5-7], and been applied successfully to the investigation of almost periodic dynamics produced by many different kinds of differential equations [8-15], and they have been some of the most attractive topics in the qualitative theory of differential equations for nearly century because of their significance and applications in areas such as physics, mathematical biology, control theory, and other related fields. As a result, several concepts were introduced as generalizations or restrictions of almost periodicity, such as asymptotic almost periodicity, pseudo-almost periodicity, weighted pseudo-almost periodicity, Stepanov-like almost periodic, Stepanov-like pseudo-almost periodic and weighted Stepanov-like pseudo-almost periodic (see, for example, [16-22]; see Table 1 and the references cited therein for more details).

In the earlier 1960s, Bochner introduced the concept of almost automorphic function [23-26] in relation to some aspects of differential geometry. The notion of almost automorphic function was introduced to avoid some assumptions of uniform convergence that arise when using almost periodic function, it is an important generalization of the classical almost periodic function. From that time the theory of almost automorphic function has been studied by numerous authors, and it also has become one of the most attractive topics in the qualitative theory of differential equations because of its significance and applications. Meanwhile, stimulated by [23-26], many interesting generalizations of the almost automorphic function have been introduced, including asymptotic almost automorphy by N'Guérékata [27], pseudo-almost automorphy by Xiao et al. [28], weighted pseudoalmost automorphy by Blot et al. [29], Stepanov-like almost automorphy by Casarino [30], Stepanov-like pseudo-almost automorphy by Diagana [31] and weighted Stepanov-like pseudo-almost automorphy by Xia and Fan [32]. The generalizations of almost automorphy follow closely a historical development very similar to that of almost periodicity and more and more general types of almost automorphy are developed (see Table 2 and the references cited therein for more details). The relationship between the various types of almost periodicity and almost automorphy is depicted in Figure 1.

In recent years, the theory of almost automorphy and its various extensions have attracted a great deal of attention of many mathematicians due to their significance and applications in physics, mathematical biology, control theory, and so on. The existence, uniqueness, and stability of almost automorphic solution have been one of the most attractive topics in the context of various kinds of abstract differential equations [33, 34], 
Table 2 Historical development of almost automorphy

\begin{tabular}{ll}
\hline Function & Original reference \\
\hline Almost automorphic (AA) & Bochner [23] \\
Asymptotic almost automorphic (AAA) & N'Guérékata [27] \\
Pseudo almost automorphic (PAA) & Xiao et al. [28] \\
Weighted pseudo-almost automorphic (WPAA) & Blot [29] \\
Stepanov-like almost automorphic (SPA) & Casarino [30] \\
Stepanov-like pseudo-almost automorphic (SPPA) & Diagana [31] \\
Weighted Stepanov-like pseudo-almost automorphic (SPWPA) & Xia and Fan [32] \\
\hline
\end{tabular}

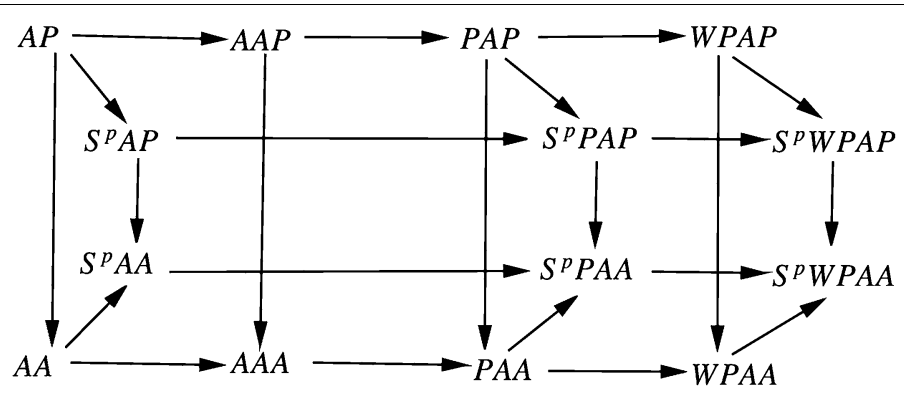

Figure 1 Relationship between almost periodic, automorphic functions, and their extensions, where ' $\rightarrow$ ' denotes the subset relation ' $c$ '.

partial differential equations [35, 36], functional differential equations [37, 38], integrodifferential equations [39] and general dynamic systems [40]. For more on these studies and related issues, we refer the reader to the references cited therein. In connection with differential equations, the great importance from both the applied and the theoretical point of view of the existence of periodic solutions is well known. However, either because models are only an approximation of reality or due to numerical errors, in practice it is impossible to verify whether a solution is exactly periodic. The concept of Stepanovlike almost automorphic function allows relaxing some assumptions to obtain solutions that have properties similar to those of a periodic function. Meanwhile, the applications of the new theory for these generalized functions, especially the Stepanov-like almost automorphic function, to various types of linear, semilinear as well as nonlinear differential equations were studied extensively (see, e.g., [29, 31, 41-53] and references therein).

In recent years, fractional differential equations have gained considerable interest due to their applications in various fields of science such as physics, mechanics, chemistry engineering etc. Significant development has been made in ordinary and partial differential equations involving fractional derivatives, we only enumerate here the monographs of Kilbas et al. [54, 55], Diethelm [56], Hilfer [57], Podlubny [58] and the papers of Agarwal et al. [59, 60], Benchohra et al. [61, 62], El-Borai [63], Lakshmikantham et al. [64-67], Mophou et al. [68-71], N'Guérékata [72], and the references therein.

Meanwhile due to their applications in fields of science where characteristics of anomalous diffusion are presented, type (1) equations are attracting increasing interest (cf. [7375 ] and references therein). For example, anomalous diffusion in fractals [74] or in macroeconomics [76] has been recently well studied in the setting of fractional Cauchy problems like (1). While the study of almost automorphic mild solutions to (1) in the borderline case $\alpha=1$ was well studied in [77, 78]. In [79] Cuevas and Lizama considered (1) when $1<\alpha<2$ and $A$ is a linear operator of sectorial negative type on a complex Banach space, 
under suitable conditions on $F$, the authors proved the existence and uniqueness of an almost automorphic mild solution to (1). Cuevas et al. [80] and [81] study, respectively, the pseudo-almost periodic and pseudo-almost periodic of class infinity mild solutions to (1) assuming that $F: \mathbb{R} \times X \rightarrow X$ is a pseudo-almost periodic and pseudo-almost periodic of class infinity functions satisfying some appropriate conditions in $x \in X$. See also [82, 83] where the $S$-asymptotically $\omega$-periodic solutions to (1) are studied. Recently, Agarwal et $a l$. [84] studied the existence and uniqueness of a weighted pseudo-almost periodic mild solution to (1), and Cao et al. [85] studied the existence of anti-periodic mild solutions to (1).

From Figure 1, we know that the weighted Stepanov-like pseudo-almost automorphic function is the most widely used function of the almost periodic type functions, and to the best of our knowledge, the existence of weighted Stepanov-like pseudo-almost automorphic mild solutions for the semilinear fractional differential equation (1) is a subject that has not been treated in the literature. Our purpose in this paper is to establish some results concerning the existence and uniqueness of weighted Stepanov-like pseudoalmost automorphic mild solutions for equations that can be modeled in the form (1). Upon making some appropriate assumptions, some sufficient conditions for the existence and uniqueness of weighted Stepanov-like pseudo-almost automorphic mild solutions to (1) are given. In particular, as application, and to illustrate our main results, we will examine some sufficient conditions for the existence and uniqueness of weighted Stepanov-like pseudo-almost automorphic mild solutions to the fractional relaxation-oscillation equation given by

$$
\partial_{t}^{\alpha} u(t, x)=\partial_{x}^{2} u(t, x)-p u(t, x)+\partial_{t}^{\alpha-1} F(t, u(t, x)), \quad t \in \mathbb{R}, x \in[0, \pi]
$$

with boundary conditions

$$
u(t, 0)=u(t, \pi)=0, \quad t \in \mathbb{R},
$$

where $F$ satisfies some additional conditions.

The rest of this paper is organized as follows. In Section 2 we recall some concepts and prove some preliminary results. The section that follows contains the main results of this paper with four existence and uniqueness theorems. In the last section, we prove the existence and uniqueness of weighted Stepanov-like pseudo-almost automorphic mild solutions for a fractional relaxation-oscillation equation as an example to illustrate our main results.

\section{Preliminaries}

We begin this section by giving some notations. Throughout this paper, let $p \in[1, \infty)$, denote by $\mathbb{N}, \mathbb{Z}$ and $\mathbb{R}$ the set of positive integers, the set of integers and the set of real numbers, respectively. Let $(X,\|\cdot\|),\left(Y,\|\cdot\|_{Y}\right)$ be two Banach spaces. Let $B C(\mathbb{R}, X)$ (respectively, $B C(\mathbb{R} \times Y, X))$ denote the space of bounded continuous functions with supremum norm

$$
\|x\|_{\infty}=\sup \{\|x(t)\|: t \in \mathbb{R}\}
$$


(respectively, the space of jointly bounded continuous functions). By $L(Y, X)$ we denote the Banach space of all bounded linear operators from $Y$ to $X$. If $Y=X$, it is simply denoted by $L(X)$.

Now, let us recall some basic definitions and results on almost automorphic functions.

Definition 2.1 (Bochner [1]) A continuous function $f: \mathbb{R} \rightarrow X$ is said to be almost automorphic if for every sequence of real numbers $\left\{s_{n}^{\prime}\right\}_{n=1}^{\infty}$, one can extract a subsequence $\left\{s_{n}\right\}_{n=1}^{\infty}$ such that

$$
g(t)=\lim _{n \rightarrow \infty} f\left(t+s_{n}\right)
$$

is well defined in $t \in \mathbb{R}$, and

$$
\lim _{n \rightarrow \infty} g\left(t-s_{n}\right)=f(t)
$$

for each $t \in \mathbb{R}$.

Denote by $A A(\mathbb{R}, X)$ the set of all such functions.

Definition 2.2 [1] A continuous function

$$
f: \mathbb{R} \times Y \rightarrow X
$$

is said to be almost automorphic if $f(t, x)$ is almost automorphic in $t \in \mathbb{R}$ uniformly for all $x \in K$, where $K$ is any bounded subset of $Y$.

Denote by $A A(\mathbb{R} \times Y, X)$ the set of all such functions.

Remark 2.1 The function $g$ in Definition 2.1 is measurable but not necessarily continuous. Moreover, if $g$ is continuous, then $f$ is uniformly continuous (cf., e.g., [86], Theorem 2.6). If the convergence in Definition 2.1 is uniform in $t \in \mathbb{R}$, then $f$ is almost periodic. A classical example of almost automorphic function (not almost periodic) is $(c f .[45,46])$

$$
f(t)=\sin \left(\frac{1}{2+\cos t+\cos \sqrt{2} t}\right), \quad t \in \mathbb{R} .
$$

Next, let us recall some definitions and basic results on Stepanov-like almost automorphic functions (for more details, see [87]).

Definition 2.3 The Bochner transform

$$
f^{b}(t, s), \quad t \in \mathbb{R}, s \in[0,1],
$$

of a function $f: \mathbb{R} \rightarrow X$ is defined by

$$
f^{b}(t, s):=f(t+s)
$$

Definition 2.4 Let $p \in[1, \infty)$. The space $B S^{p}(\mathbb{R}, X)$ of all Stepanov bounded functions, with the exponent $p$, consists of all measurable functions $f: \mathbb{R} \rightarrow X$ such that

$$
f^{b} \in L^{\infty}\left(\mathbb{R}, L^{p}([0,1], X)\right) .
$$


This is a Banach space with the norm

$$
\|f\|_{S^{p}}:=\left\|f^{b}\right\|_{L^{\infty}\left(\mathbb{R}, L^{p}\right)}=\sup _{t \in \mathbb{R}}\left(\int_{t}^{t+1}\|f(\tau)\|^{p} \mathrm{~d} \tau\right)^{\frac{1}{p}}
$$

Definition 2.5 The space $S^{p} A A(\mathbb{R}, X)$ of Stepanov-like almost automorphic functions consists of all $f \in B S^{p}(\mathbb{R}, X)$ such that

$$
f^{b} \in A A\left(\mathbb{R}, L^{p}([0,1], X)\right) .
$$

That is, a function $f \in L_{\text {loc }}^{p}(\mathbb{R}, X)$ is said to be Stepanov-like almost automorphic if its Bochner transform

$$
f^{b}: \mathbb{R} \rightarrow L^{p}([0,1], X)
$$

is almost automorphic in the sense that for every sequence of real numbers $\left\{s_{n}^{\prime}\right\}_{n=1}^{\infty}$, there exist a subsequence $\left\{s_{n}\right\}_{n=1}^{\infty}$ and a function $g \in L_{\text {loc }}^{p}(\mathbb{R}, X)$ such that

$$
\left[\int_{0}^{1}\left\|f\left(t+s+s_{n}\right)-g(t+s)\right\|^{p} \mathrm{~d} s\right]^{\frac{1}{p}} \rightarrow 0,
$$

and

$$
\left[\int_{0}^{1}\left\|g\left(t+s-s_{n}\right)-f(t+s)\right\|^{p} \mathrm{~d} s\right]^{\frac{1}{p}} \rightarrow 0,
$$

as $n \rightarrow \infty$ for all $t \in \mathbb{R}$.

Definition 2.6 A function

$$
f: \mathbb{R} \times Y \rightarrow X, \quad(t, x) \rightarrow f(t, x)
$$

with

$$
f(\cdot, x) \in L_{\mathrm{loc}}^{p}(\mathbb{R}, X)
$$

for each $x \in Y$ is said to be Stepanov-like almost automorphic in $t \in \mathbb{R}$ uniformly for $x \in Y$, if $t \rightarrow f(t, x)$ is Stepanov-like almost automorphic for each $x \in Y$. That is, for every sequence of real numbers $\left\{s_{n}^{\prime}\right\}_{n=1}^{\infty}$, there exist a subsequence $\left\{s_{n}\right\}_{n=1}^{\infty}$ and a function

$$
g(\cdot, x) \in L_{\mathrm{loc}}^{p}(\mathbb{R}, X)
$$

such that

$$
\left[\int_{0}^{1}\left\|f\left(t+s+s_{n}, x\right)-g(t+s, x)\right\|^{p} \mathrm{~d} s\right]^{\frac{1}{p}} \rightarrow 0,
$$


and

$$
\left[\int_{0}^{1}\left\|g\left(t+s-s_{n}, x\right)-f(t+s, x)\right\|^{p} \mathrm{~d} s\right]^{\frac{1}{p}} \rightarrow 0
$$

as $n \rightarrow \infty$ for all $t \in \mathbb{R}$ and $x \in Y$.

Denote by $S^{p} A A(\mathbb{R} \times Y, X)$ the set of all such functions.

Remark 2.2 It is clear that, if $x: \mathbb{R} \rightarrow X$ is an almost automorphic function, then $x$ is a Stepanov-like almost automorphic function, that is,

$$
A A(\mathbb{R}, X) \subset S^{p} A A(\mathbb{R}, X) .
$$

Let $U$ be the set of all functions $\rho: \mathbb{R} \rightarrow[0, \infty)$ which are positive and locally integrable over $\mathbb{R}$. For a given $r>0$ and each $\rho \in U$, set

$$
m(r, \rho):=\int_{-r}^{r} \rho(x) \mathrm{d} x
$$

and the notation $U_{\infty}$ stands for the set of weight functions

$$
U_{\infty}:=\left\{\rho \in U: \lim _{r \rightarrow \infty} m(r, \rho)=\infty\right\}
$$

For $\rho \in U_{\infty}$, define the weighted ergodic space

$$
\begin{aligned}
& P A A_{0}(\mathbb{R}, X, \rho):=\left\{\varphi \in B C(\mathbb{R}, X): \lim _{r \rightarrow \infty} \frac{1}{m(r, \rho)} \int_{-r}^{r}\|\varphi(t)\| \rho(t) \mathrm{d} t=0\right\}, \\
& P A A_{0}(\mathbb{R} \times Y, X, \rho) \\
& :=\{\varphi \in C(\mathbb{R} \times Y, X): \varphi(\cdot, x) \text { is bounded for each } x \in Y \text { and } \\
& \left.\quad \lim _{r \rightarrow \infty} \frac{1}{m(r, \rho)} \int_{-r}^{r}\|\varphi(t, x)\| \rho(t) \mathrm{d} t=0 \text { uniformly in compact subset of } Y\right\} .
\end{aligned}
$$

Definition 2.7 [32] Let $\rho \in U_{\infty}$. A continuous function

$$
f \in B S^{p}(\mathbb{R}, X)
$$

is said to be weighted Stepanov-like pseudo-almost automorphic (or weighted $S^{p}$-pseudoalmost automorphic) if it can be decomposed as

$$
f=g+\varphi
$$

where

$$
g \in S^{p} A A(\mathbb{R}, X), \quad \varphi \in P A A_{0}\left(\mathbb{R}, L^{p}([0,1], X), \rho\right) .
$$

In other words, a function

$$
f \in L_{\mathrm{loc}}^{p}(\mathbb{R}, X)
$$


is said to be weighted Stepanov-like pseudo-almost automorphic relatively to the weight $\rho \in U_{\infty}$, if its Bochner transform

$$
f^{b}: \mathbb{R} \rightarrow L^{p}([0,1], X)
$$

is weighted pseudo-almost automorphic in the sense that there exist two functions $g, \varphi$ : $\mathbb{R} \rightarrow X$ such that

$$
f=g+\varphi
$$

where

$$
g^{b} \in A A(\mathbb{R}, X), \quad \varphi \in P A A_{0}\left(\mathbb{R}, L^{p}([0,1], X), \rho\right) .
$$

We denote by $S^{p} W P A A(\mathbb{R}, X)$ the set of all such functions.

Definition 2.8 [32] Let $\rho \in U_{\infty}$. A function

$$
f: \mathbb{R} \times Y \rightarrow X, \quad(t, x) \rightarrow f(t, x)
$$

with

$$
f(\cdot, x) \in L_{\mathrm{loc}}^{p}(\mathbb{R}, X)
$$

for each $x \in Y$ is said to be weighted Stepanov-like pseudo-almost automorphic (or $S^{p}$ weighted pseudo-almost automorphic) if it can be expressed as

$$
f=g+\varphi,
$$

where

$$
g \in S^{p} A A(\mathbb{R} \times Y, X), \quad \varphi \in P A A_{0}\left(\mathbb{R} \times Y, L^{p}([0,1], X), \rho\right) .
$$

We denote by $S^{p} W P A A(\mathbb{R} \times Y, X)$ the set of all such functions.

Now we give some lemmas for weighted Stepanov-like pseudo-almost automorphic functions.

Lemma 2.1 [32] Let $\rho \in U_{\infty}$. Assume that

$$
P A A_{0}\left(\mathbb{R}, L^{p}([0,1], X), \rho\right)
$$

is translation invariant. Then the decomposition of a $S^{p}$-weighted pseudo-almost automorphic function is unique.

Lemma $2.2[32] S^{q} W P A A(\mathbb{R}, X, \rho) \subset S^{p} W P A A(\mathbb{R}, X, \rho)$ for $1 \leq p<q<+\infty$. 
Lemma 2.3 [32] Assume that

$$
f, f_{1}, f_{2} \in S^{p} W P A A(\mathbb{R}, X, \rho), \quad \rho \in U_{\infty} .
$$

Then

(i) $f_{1}+f_{2} \in S^{p} W P A A(\mathbb{R}, X, \rho)$.

(ii) $\lambda f \in S^{p} W P A A(\mathbb{R}, X, \rho)$ for any $\lambda \in \mathbb{R}$.

(iii) If

$$
\limsup _{t \rightarrow \infty} \frac{\rho(t+\tau)}{\rho(t)} \text { and } \quad \limsup _{T \rightarrow \infty} \frac{m(T+|\tau|, \rho)}{m(T, \rho)}
$$

are finite for $\tau \in \mathbb{R}$, then

$$
f(t-\tau) \in S^{p} W P A A(\mathbb{R}, X, \rho) .
$$

Lemma 2.4 [32] Let $\rho \in U_{\infty}$. The space $S^{p} W P A A(\mathbb{R}, X, \rho)$ equipped with the norm $\|\cdot\|_{S^{p}}$ is a Banach space.

Lemma 2.5 [32] Assume that $\rho \in U_{\infty}$,

$$
f=g+\varphi \in S^{p} W P A A(\mathbb{R} \times X, X, \rho)
$$

with

$$
g^{b} \in A A\left(\mathbb{R} \times X, L^{p}([0,1], X)\right), \quad \varphi \in P A A_{0}\left(\mathbb{R} \times X, L^{p}([0,1], X)\right),
$$

and:

(i) There exist constants $L_{f}, L_{g}>0$ such that

$$
\|f(t, x)-f(t, y)\| \leq L_{f}\|x-y\|, \quad\|g(t, x)-g(t, y)\| \leq L_{g}\|x-y\|, \quad x, y \in X, t \in \mathbb{R} .
$$

(ii) $h=\alpha+\beta \in S^{p} W P A A(\mathbb{R}, X, \rho)$ with

$$
\alpha^{b} \in A A\left(\mathbb{R}, L^{p}([0,1], X)\right), \quad \varphi \in P A A_{0}\left(\mathbb{R}, L^{p}([0,1], X)\right),
$$

and

$$
K=\overline{\{\alpha(t): t \in \mathbb{R}\}}
$$

is compact in $X$.

Then

$$
f(\cdot, h(\cdot)) \in S^{p} \operatorname{WPAA}(\mathbb{R}, X, \rho) .
$$

Lemma 2.6 [32] Assume that $\rho \in U_{\infty}$,

$$
f=g+\varphi \in S^{p} W P A A(\mathbb{R} \times X, X, \rho)
$$


with

$$
g^{b} \in A A\left(\mathbb{R} \times X, L^{p}([0,1], X)\right), \quad \varphi \in P A A_{0}\left(\mathbb{R} \times X, L^{p}([0,1], X)\right),
$$

and:

(i) There exist nonnegative functions

$$
L_{f}, L_{g} \in S^{r} A A(\mathbb{R}, \mathbb{R})
$$

with

$$
r \geq \max \left\{p, \frac{p}{p-1}\right\}
$$

such that

$$
\|f(t, x)-f(t, y)\| \leq L_{f}(t)\|x-y\|, \quad\|g(t, x)-g(t, y)\| \leq L_{g}(t)\|x-y\|, \quad x, y \in X, t \in \mathbb{R} .
$$

(ii) $h=\alpha+\beta \in S^{p} W P A A(\mathbb{R}, X, \rho)$ with

$$
\alpha^{b} \in A A\left(\mathbb{R}, L^{p}([0,1], X)\right), \quad \varphi \in P A A_{0}\left(\mathbb{R}, L^{p}([0,1], X)\right),
$$

and

$$
K=\overline{\{\alpha(t): t \in \mathbb{R}\}}
$$

is compact in $X$.

Then there exists $q \in[1, p)$ such that

$$
f(\cdot, h(\cdot)) \in S^{p} W P A A(\mathbb{R}, X, \rho) .
$$

Now we give a lemma.

Lemma 2.7 Let $\left\{x_{n}(t)\right\}_{n \in \mathbb{N}}$ be a sequence of Stepanov-like pseudo-almost automorphic functions such that

$$
\int_{0}^{1}\left\|x_{n}(t+s)-x(t+s)\right\|^{p} \mathrm{~d} s \rightarrow 0,
$$

as $n \rightarrow \infty$ for each $t \in \mathbb{R}$, then

$$
x \in S^{p} A A(\mathbb{R}, X) .
$$

Proof For any $i \in \mathbb{N}$ fixed, since

$$
x_{i}(t) \in S^{p} A A(\mathbb{R}, X),
$$


for every sequence of real numbers $\left\{s_{n}^{\prime}\right\}_{n \in \mathbb{N}}$, there exist a subsequence $\left\{s_{n}\right\}_{n \in \mathbb{N}}$ and a function $y_{i} \in L_{\mathrm{loc}}^{p}(\mathbb{R}, X)$ such that

$$
\begin{aligned}
& {\left[\int_{0}^{1}\left\|x_{i}\left(t+s_{n}+s\right)-y_{i}(t+s)\right\|^{p} \mathrm{~d} s\right]^{\frac{1}{p}} \rightarrow 0,} \\
& {\left[\int_{0}^{1}\left\|y_{i}\left(t-s_{n}+s\right)-x_{i}(t+s)\right\|^{p} \mathrm{~d} s\right]^{\frac{1}{p}} \rightarrow 0,}
\end{aligned}
$$

as $n \rightarrow \infty$ for all $t \in \mathbb{R}$. On the other hand, from (2), one can easily deduce that $\left\{x_{n}(t)\right\}_{n \in \mathbb{N}}$ is a Cauchy sequence with respect to $\|\cdot\|_{S^{p}}$. Observe that, for each $t \in \mathbb{R}$, the sequence $y_{i}$ is also a Cauchy sequence in $L_{\mathrm{loc}}^{p}(\mathbb{R}, X)$. Indeed, if we write

$$
y_{i}(t)-y_{j}(t)=y_{i}(t)-x_{i}\left(t+s_{n}\right)+x_{i}\left(t+s_{n}\right)-x_{j}\left(t+s_{n}\right)+x_{j}\left(t+s_{n}\right)-y_{j}(t),
$$

then for a sufficiently large $n$, one gets

$$
\begin{aligned}
& {\left[\int_{0}^{1}\left\|y_{i}(t+s)-y_{j}(t+s)\right\|^{p} \mathrm{~d} s\right]^{\frac{1}{p}}} \\
& \quad \leq\left[\int _ { 0 } ^ { 1 } \left(\left\|y_{i}(t+s)-x_{i}\left(t+s+s_{n}\right)\right\|+\left\|x_{i}\left(t+s+s_{n}\right)-x_{j}\left(t+s+s_{n}\right)\right\|\right.\right. \\
& \left.\left.\quad+\left\|x_{j}\left(t+s+s_{n}\right)-y_{j}(t+s)\right\|\right)^{p} \mathrm{~d} s\right]^{\frac{1}{p}} \\
& \quad 3\left[\int _ { 0 } ^ { 1 } \left(\left\|y_{i}(t+s)-x_{i}\left(t+s+s_{n}\right)\right\|^{p}+\left\|x_{i}\left(t+s+s_{n}\right)-x_{j}\left(t+s+s_{n}\right)\right\|^{p}\right.\right. \\
& \left.\left.\quad+\left\|x_{j}\left(t+s+s_{n}\right)-y_{j}(t+s)\right\|^{p}\right) \mathrm{~d} s\right]^{\frac{1}{p}} .
\end{aligned}
$$

By (2), (3), and (4), the sequence of $y_{i}$ is a Cauchy sequence in $L_{\mathrm{loc}}^{p}(\mathbb{R}, X)$.

Using the completeness of $L_{\mathrm{loc}}^{p}(\mathbb{R}, X)$, we denote by $y(t)$ the pointwise limit of $y_{i}(t)$. Now let us prove that

$$
x(t) \in S^{p} A A(\mathbb{R}, X) .
$$

Note that the inequality below holds for any index $i$ and any $t \in \mathbb{R}$,

$$
\begin{aligned}
& {\left[\int_{0}^{1}\left\|x\left(t+s+s_{n}\right)-y(t+s)\right\|^{p} \mathrm{~d} s\right]^{\frac{1}{p}}} \\
& \leq\left[\int _ { 0 } ^ { 1 } \left(\left\|x\left(t+s+s_{n}\right)-x_{i}\left(t+s+s_{n}\right)\right\|\right.\right. \\
& \left.\left.\quad+\left\|x_{i}\left(t+s+s_{n}\right)-y_{i}(t+s)\right\|+\left\|y_{i}(t+s)-y(t+s)\right\|\right)^{p} \mathrm{~d} s\right]^{\frac{1}{p}} \\
& \leq 3\left[\int _ { 0 } ^ { 1 } \left(\left\|x\left(t+s+s_{n}\right)-x_{i}\left(t+s+s_{n}\right)\right\|^{p}+\left\|x_{i}\left(t+s+s_{n}\right)-y_{i}(t+s)\right\|^{p}\right.\right. \\
& \left.\left.\quad+\left\|y_{i}(t+s)-y(t+s)\right\|^{p}\right) \mathrm{~d} s\right]^{\frac{1}{p}} .
\end{aligned}
$$


So, from (2) and the fact that $y(t)$ is the pointwise limit of $y_{i}(t)$, for any sufficiently small $\varepsilon>0$ there exists a sufficiently large $i$, such that for each $t \in \mathbb{R}$,

$$
\begin{aligned}
& \int_{0}^{1}\left\|x_{i}\left(t+s+s_{n}\right)-y_{i}(t+s)\right\|^{p} \mathrm{~d} s<\frac{\varepsilon^{p}}{3^{p+1}}, \\
& \int_{0}^{1}\left\|x\left(t+s+s_{n}\right)-x_{i}\left(t+s+s_{n}\right)\right\|^{p} \mathrm{~d} s<\frac{\varepsilon^{p}}{3^{p+1}} .
\end{aligned}
$$

Now for this sufficiently large $i$, from (3) and (4), there exists a sufficient $N$ such that for any $n>N$ one has

$$
\int_{0}^{1}\left\|y_{i}(t+s)-y(t+s)\right\|^{p} \mathrm{~d} s<\frac{\varepsilon^{p}}{3^{p+1}} .
$$

Thus

$$
\left[\int_{0}^{1}\left\|x\left(t+s+s_{n}\right)-y(t+s)\right\|^{p} \mathrm{~d} s\right]^{\frac{1}{p}}<\varepsilon, \quad \text { for } n>N,
$$

which implies

$$
\left[\int_{0}^{1}\left\|x\left(t+s+s_{n}\right)-y(t+s)\right\|^{p} \mathrm{~d} s\right]^{\frac{1}{p}} \rightarrow 0
$$

as $n \rightarrow \infty$ pointwise on $\mathbb{R}$. One can use the same steps to prove that

$$
\left[\int_{0}^{1}\left\|y\left(t+s-s_{n}\right)-x(t+s)\right\|^{p} \mathrm{~d} s\right]^{\frac{1}{p}} \rightarrow 0,
$$

as $n \rightarrow \infty$ pointwise on $\mathbb{R}$. That is,

$$
x(t) \in S^{p} A A(\mathbb{R}, X) .
$$

The proof is finished.

We need some basic definitions and properties of the fractional calculus theory which are used further in this paper.

Definition 2.9 [54] The fractional Riemann-Liouville integral of order $\alpha>0$ with the lower limit $t_{0}$ for a function $f$ is defined as

$$
I^{\alpha} f(t)=\frac{1}{\Gamma(\alpha)} \int_{t_{0}}^{t}(t-s)^{\alpha-1} f(s) \mathrm{d} s, \quad t>t_{0}, \alpha>0
$$

provided the right-hand side is pointwise defined on $\left[t_{0}, \infty\right)$, where $\Gamma$ is the Gamma function. 
Definition 2.10 [54] The Riemann-Liouville derivative of order $\alpha>0$ with the lower limit $t_{0}$ for a function $f:\left[t_{0}, \infty\right) \rightarrow \mathbb{R}$ can be written as

$$
D_{t}^{\alpha} f(t)=\frac{1}{\Gamma(n-\alpha)} \frac{\mathrm{d}^{n}}{\mathrm{~d} t^{n}} \int_{t_{0}}^{t}(t-s)^{-\alpha} f(s) \mathrm{d} s, \quad t>t_{0}, n-1<\alpha<n .
$$

Remark 2.3 The first and maybe the most important property of the Riemann-Liouville fractional derivative is that, for $t>t_{0}$ and $\alpha>0$, one has

$$
D_{t}^{\alpha}\left(I^{\alpha} f(t)\right)=f(t)
$$

which means that the Riemann-Liouville fractional differentiation operator is a left inverse to the Riemann-Liouville fractional integration operator of the same order $\alpha$.

In the following, we give the definitions of sectorial linear operators and their associated solution operators.

Recall that a closed and linear operator $A$ is said to be sectorial of type $\omega$ and angle $\theta$ if there exist

$$
0<\theta<\frac{\pi}{2}, \quad M>0, \quad \omega \in \mathbb{R},
$$

such that its resolvent exists outside the sector

$$
\omega+S_{\theta}:=\{\omega+\lambda: \lambda \in \mathbb{C},|\arg (-\lambda)|<\theta\},
$$

and

$$
\left\|(\lambda-A)^{-1}\right\| \leq \frac{M}{|\lambda-\omega|}, \quad \lambda \notin \omega+S_{\theta} .
$$

Sectorial operators are well studied in the literature, usually for the case $\omega=0$. For a recent reference including several examples and properties we refer the reader to [88]. Note that an operator $A$ is sectorial of type $\omega$ if and only if $\omega I-A$ is sectorial of type 0 .

Definition 2.11 [89] Let $A$ be a closed and linear operator with domain $D(A)$ defined on a Banach space $X$. We call $A$ is the generator of a solution operator if there are $\omega \in \mathbb{R}$ and a strongly continuous function

$$
S_{\alpha}: \mathbb{R}^{+} \rightarrow L(X)
$$

such that

$$
\left\{\lambda^{\alpha}: \operatorname{Re} \lambda>\omega\right\} \subseteq \rho(A)
$$

and

$$
\lambda^{\alpha-1}\left(\lambda^{\alpha}-A\right)^{-1} x=\int_{0}^{\infty} e^{-\lambda t} S_{\alpha}(t) x \mathrm{~d} t, \quad \operatorname{Re} \lambda>\omega, x \in X .
$$

In this case, $S_{\alpha}(t)$ is called the solution operator generated by $A$. 
We note that if $A$ is sectorial of type $\omega$ with

$$
0 \leq \theta \leq \pi\left(1-\frac{\alpha}{2}\right)
$$

then $A$ is the generator of a solution operator given by

$$
S_{\alpha}(t):=\frac{1}{2 \pi i} \int_{\gamma} e^{-\lambda t} \lambda^{\alpha-1}\left(\lambda^{\alpha}-A\right)^{-1} \mathrm{~d} \lambda,
$$

where $\gamma$ is a suitable path lying outside the sector $\omega+\Sigma_{\theta}$ (cf. [88]).

Very recently, Cuesta in [89], Theorem 1, has proved that if $A$ is a sectorial operator of type $\omega<0$ for some $M>0$ and

$$
0 \leq \theta<\pi\left(1-\frac{\alpha}{2}\right)
$$

then there exists $C>0$ such that

$$
\left\|S_{\alpha}(t)\right\|_{L(X)} \leq \frac{C M}{1+|\omega| t^{\alpha}},
$$

for $t \geq 0$. In the border case $\alpha=1$, this is analogous to saying that $A$ is the generator of an exponentially stable $C_{0}$-semigroup. The main difference is that in the case $\alpha>1$ the solution family $S_{\alpha}(t)$ decays like $t^{-\alpha}$. Cuesta's result proves that $S_{\alpha}(t)$ is, in fact, integrable.

Now we give another lemma.

Lemma 2.8 Assume that (6) is true. Given a function

$$
F(t) \in S^{p} W P A A(\mathbb{R}, X) .
$$

Let

$$
[\Phi F](t):=\int_{-\infty}^{t} S_{\alpha}(t-s) F(s) \mathrm{d} s
$$

Then $[\Phi F](t)$ is weighted Stepanov-like pseudo-almost automorphic.

Proof Firstly, note that

$$
\int_{0}^{\infty} \frac{1}{1+|\omega| s^{\alpha}} \mathrm{d} s=\frac{\omega^{-\frac{1}{\alpha}} \pi}{\alpha \sin \frac{\pi}{\alpha}} \quad \text { for } 1<\alpha<2
$$

By condition (6), one has

$$
\begin{aligned}
& {\left[\int_{t}^{t+1}\left\|\int_{-\infty}^{\sigma} S_{\alpha}(\sigma-s) F(s) \mathrm{d} s\right\|^{p} \mathrm{~d} \sigma\right]^{\frac{1}{p}}} \\
& \quad=\left[\int_{t}^{t+1}\left\|\int_{0}^{\infty} S_{\alpha}(\tau) F(\sigma-\tau) \mathrm{d} \tau\right\|^{p} \mathrm{~d} \sigma\right]^{\frac{1}{p}}
\end{aligned}
$$




$$
\begin{aligned}
& \leq C M\left[\int_{t}^{t+1} \int_{0}^{\infty}\left(\frac{1}{1+|\omega| \tau^{\alpha}}\right)^{p}\|F(\sigma-\tau)\|^{p} \mathrm{~d} \tau \mathrm{d} \sigma\right]^{\frac{1}{p}} \\
& \leq C M\|F\|_{S^{p}}\left(\int_{0}^{\infty}\left(\frac{1}{1+|\omega| \tau^{\alpha}}\right)^{p} \mathrm{~d} \tau\right)^{\frac{1}{p}} \\
& \leq C M\|F\|_{S^{p}}\left(\int_{0}^{\infty} \frac{1}{1+|\omega| \tau^{\alpha}} \mathrm{d} \tau\right)^{\frac{1}{p}} \\
& =C M\|F\|_{S^{p}}\left[\frac{\omega^{-\frac{1}{\alpha}} \pi}{\alpha \sin \frac{\pi}{\alpha}}\right]^{\frac{1}{p}} .
\end{aligned}
$$

Thus, $\Phi$ is well defined and $\Phi F$ is bounded. On the other hand, for any $t, h \in \mathbb{R}$,

$$
\begin{aligned}
& \left(\int_{t}^{t+1}\|[\Phi F](\sigma+h)-[\Phi F](\sigma)\|^{p} \mathrm{~d} \sigma\right)^{\frac{1}{p}} \\
& \quad=\left(\int_{t}^{t+1}\left\|\int_{-\infty}^{\sigma+h} S_{\alpha}(\sigma+h-s) F(s) \mathrm{d} s-\int_{-\infty}^{\sigma} S_{\alpha}(\sigma-s) F(s) \mathrm{d} s\right\|^{p} \mathrm{~d} \sigma\right)^{\frac{1}{p}} \\
& \quad=\left(\int_{t}^{t+1}\left\|\int_{-\infty}^{\sigma} S_{\alpha}(\sigma-s)[F(s+h)-F(s)] \mathrm{d} s\right\|^{p} \mathrm{~d} \sigma\right)^{\frac{1}{p}} \\
& \quad=\left(\int_{t}^{t+1}\left\|\int_{0}^{\infty} S_{\alpha}(\tau)[F(\sigma-\tau+h)-F(\sigma-\tau)] \mathrm{d} \tau\right\|^{p} \mathrm{~d} \sigma\right)^{\frac{1}{p}} \\
& \quad \leq C M\|F(t+h)-F(t)\|_{S^{p}}\left(\int_{0}^{\infty}\left[\frac{1}{1+|\omega| \tau^{\alpha}}\right]^{p} \mathrm{~d} \tau\right)^{\frac{1}{p}} \\
& \quad \leq C M\|F(t+h)-F(t)\|_{S^{p}}\left(\int_{0}^{\infty} \frac{1}{1+|\omega| \tau^{\alpha}} \mathrm{d} \tau\right)^{\frac{1}{p}} \\
& \quad=C M\left[\frac{\omega^{-\frac{1}{\alpha}} \pi}{\alpha \sin \frac{\pi}{\alpha}}\right]^{\frac{1}{p}}\|F(t+h)-F(t)\|_{S^{p}},
\end{aligned}
$$

which shows that $\Phi F$ is continuous. Since

$$
F \in S^{p} W P A A(\mathbb{R}, X)
$$

there exist

$$
G \in S^{p} A A(\mathbb{R}, X) \quad \text { and } \quad \Phi \in P A A_{0}\left(\mathbb{R}, L^{p}([0,1], X), \rho\right),
$$

such that $F=G+\Phi$. So

$$
\begin{aligned}
x(t) & =\int_{-\infty}^{t} S_{\alpha}(t-\sigma) F(\sigma) \mathrm{d} \sigma=\int_{-\infty}^{t} S_{\alpha}(t-\sigma) G(\sigma) \mathrm{d} \sigma+\int_{-\infty}^{t} S_{\alpha}(t-\sigma) \Phi(\sigma) \mathrm{d} \sigma \\
& =\Xi_{1}(t)+\Xi_{2}(t) .
\end{aligned}
$$

We only need to verify

$$
\Xi_{1}(t) \in S^{p} A A(\mathbb{R}, X), \quad \Xi_{2}(t) \in P A A_{0}(\mathbb{R}, X, \rho) .
$$


First we prove that

$$
\Xi_{1}(t) \in S^{p} A A(\mathbb{R}, X) .
$$

Let $\left\{s_{m}^{\prime}\right\}_{m \in \mathbb{N}}$ be a sequence of real numbers. Since

$$
G \in S^{p} A A(\mathbb{R}, X),
$$

there exist a subsequence $\left\{s_{m}\right\}_{m \in \mathbb{N}}$ of $\left\{s_{m}^{\prime}\right\}_{m \in \mathbb{N}}$ and a function $\tilde{G}$ such that

$$
\begin{aligned}
& {\left[\int_{0}^{1}\left\|G\left(t+s_{m}+s\right)-\tilde{G}(t+s)\right\|^{p} \mathrm{~d} s\right]^{\frac{1}{p}} \rightarrow 0,} \\
& {\left[\int_{0}^{1}\left\|\tilde{G}\left(t-s_{m}+s\right)-G(t+s)\right\|^{p} \mathrm{~d} s\right]^{\frac{1}{p}} \rightarrow 0,}
\end{aligned}
$$

as $m \rightarrow \infty$ pointwise on $\mathbb{R}$ for each $x \in X$. Let

$$
[\Phi \tilde{G}](t):=\int_{-\infty}^{t} S_{\alpha}(t-s) \tilde{G}(s) \mathrm{d} s .
$$

Thus

$$
\begin{aligned}
& \left(\int_{0}^{1}\left\|[\Phi G]\left(t+s+s_{m}\right)-[\Phi \tilde{G}](t+s)\right\|^{p} \mathrm{~d} s\right)^{\frac{1}{p}} \\
& \quad=\left(\int_{0}^{1}\left\|\int_{-\infty}^{t} S_{\alpha}(\sigma) G\left(t+s+s_{m}-\sigma\right) \mathrm{d} \sigma-\int_{-\infty}^{t} S_{\alpha}(\sigma) \tilde{G}(t+s-\sigma) \mathrm{d} \sigma\right\|^{p} \mathrm{~d} s\right)^{\frac{1}{p}} \\
& \quad=\left(\int_{0}^{1}\left\|\int_{-\infty}^{t} S_{\alpha}(\sigma)\left[G\left(t+s+s_{m}-\sigma\right)-\tilde{G}(t+s-\sigma)\right] \mathrm{d} \sigma\right\|^{p} \mathrm{~d} s\right)^{\frac{1}{p}} \\
& \quad \leq\left(\int_{0}^{1}\left(\int_{-\infty}^{t}\left\|S_{\alpha}(\sigma)\right\|\left\|G\left(t+s+s_{m}-\sigma\right)-\tilde{G}(t+s-\sigma)\right\| \mathrm{d} \sigma\right)^{p} \mathrm{~d} s\right)^{\frac{1}{p}} \\
& \quad \leq\left(\int_{0}^{1} \int_{-\infty}^{t}\left\|S_{\alpha}(\sigma)\right\|^{p}\left\|G\left(t+s+s_{m}-\sigma\right)-\tilde{G}(t+s-\sigma)\right\|^{p} \mathrm{~d} \sigma \mathrm{d} s\right)^{\frac{1}{p}} \\
& \quad \leq\left(\int_{0}^{1} \int_{0}^{\infty}\left[\frac{C M}{1+|\omega| \sigma^{\alpha}}\right]^{p}\left\|G\left(t+s+s_{m}-\sigma\right)-\tilde{G}(t+s-\sigma)\right\|^{p} \mathrm{~d} \sigma \mathrm{d} s\right)^{\frac{1}{p}} \\
& \quad=\left(\int_{0}^{\infty}\left[\frac{C M}{1+|\omega| \sigma^{\alpha}}\right]^{p} \int_{0}^{1}\left\|G\left(t+s+s_{m}-\sigma\right)-\tilde{G}(t+s-\sigma)\right\|^{p} \mathrm{~d} s \mathrm{~d} \sigma\right)^{\frac{1}{p}} \\
& \leq\left(\int_{0}^{\infty}\left[\frac{C^{p} M^{p}}{1+|\omega| \sigma^{\alpha}}\right] \int_{0}^{1}\left\|G\left(t+s+s_{m}-\sigma\right)-\tilde{G}(t+s-\sigma)\right\|^{p} \mathrm{~d} s \mathrm{~d} \sigma\right)^{\frac{1}{p}} .
\end{aligned}
$$

From (7), (8), and (9), obviously, the last inequality goes to 0 as $m \rightarrow \infty$ pointwise on $\mathbb{R}$. Similarly one can prove that

$$
\left[\int_{0}^{1}\left\|[\Phi \tilde{G}]\left(t+s-s_{m}\right)-[\Phi G](t+s)\right\|^{p} \mathrm{~d} s\right]^{\frac{1}{p}} \rightarrow 0
$$


as $m \rightarrow \infty$ pointwise on $\mathbb{R}$. Thus we conclude that

$$
[\Phi G] \in S^{p} A A(\mathbb{R}, X) \text {. }
$$

In the following, we prove that

$$
\Xi_{2}(t) \in P A A_{0}(\mathbb{R}, X, \rho) .
$$

To complete the proof, consider for each $n=1,2, \ldots$, the integrals

$$
\Theta_{n}(t)=\int_{t-n}^{t-n+1} S_{\alpha}(t-\sigma) \Phi(\sigma) \mathrm{d} \sigma
$$

for each $t \in \mathbb{R}$. Note that

$$
\Theta_{n}(t)=\int_{t-n}^{t-n+1} S_{\alpha}(t-\sigma) \Phi(\sigma) \mathrm{d} \sigma=\int_{n-1}^{n} S_{\alpha}(\sigma) \Phi(t-\sigma) \mathrm{d} \sigma,
$$

and by using the Hölder inequality, one gets

$$
\begin{aligned}
& \left\|\Theta_{n}(t)\right\|_{S^{p}}=\sup _{t \in \mathbb{R}}\left(\int_{t}^{t+1}\left\|\Theta_{n}(\tau)\right\|^{p} \mathrm{~d} \tau\right)^{\frac{1}{p}} \\
& =\sup _{t \in \mathbb{R}}\left(\int_{0}^{1}\left\|\Theta_{n}(t+s)\right\|^{p} \mathrm{~d} s\right)^{\frac{1}{p}} \\
& =\sup _{t \in \mathbb{R}}\left(\int_{0}^{1}\left\|\Theta_{n}(t+s)\right\|^{p} \mathrm{~d} s\right)^{\frac{1}{p}} \\
& =\sup _{t \in \mathbb{R}}\left(\int_{0}^{1}\left\|\int_{n-1}^{n} S_{\alpha}(\sigma) \Phi(t+s-\sigma) \mathrm{d} \sigma\right\|^{p} \mathrm{~d} s\right)^{\frac{1}{p}} \\
& \leq \sup _{t \in \mathbb{R}}\left(\int_{0}^{1}\left(\int_{n-1}^{n}\left\|S_{\alpha}(\sigma)\right\|\|\Phi(t+s-\sigma)\| \mathrm{d} \sigma\right)^{p} \mathrm{~d} s\right)^{\frac{1}{p}} \\
& \leq \sup _{t \in \mathbb{R}}\left(\int_{0}^{1} \int_{n-1}^{n}\left\|S_{\alpha}(\sigma)\right\|^{p}\|\Phi(t+s-\sigma)\|^{p} \mathrm{~d} \sigma \mathrm{d} s\right)^{\frac{1}{p}} \\
& \leq \sup _{t \in \mathbb{R}}\left(\int_{0}^{1} \int_{n-1}^{n}\left[\frac{C M}{1+|\omega| \sigma^{\alpha}}\right]^{p}\|\Phi(t+s-\sigma)\|^{p} \mathrm{~d} \sigma \mathrm{d} s\right)^{\frac{1}{p}} \\
& =\sup _{t \in \mathbb{R}}\left(\int_{n-1}^{n}\left[\frac{C M}{1+|\omega| \sigma^{\alpha}}\right]^{p} \int_{0}^{1}\|\Phi(t+s-\sigma)\|^{p} \mathrm{~d} s \mathrm{~d} \sigma\right)^{\frac{1}{p}} \\
& =\sup _{t \in \mathbb{R}}\left(\int_{n-1}^{n}\left[\frac{C M}{1+|\omega| \sigma^{\alpha}}\right]^{p}\|\Phi\|_{S^{p}}^{p} \mathrm{~d} \sigma\right)^{\frac{1}{p}} \\
& =C M\|\Phi\|_{S^{p}}\left(\int_{n-1}^{n}\left[\frac{1}{1+|\omega| \sigma^{\alpha}}\right]^{p} \mathrm{~d} \sigma\right)^{\frac{1}{p}} \\
& \leq C M\|\Phi\|_{S^{p}} \frac{1}{1+|\omega|(n-1)^{\alpha}} \\
& \leq \frac{C M\|\Phi\|_{S^{p}}}{|\omega|} \frac{1}{(n-1)^{\alpha}} \text {. }
\end{aligned}
$$


From $1<\alpha<2$, it follows that

$$
\frac{C M\|\Phi\|_{S^{p}}^{p}}{|\omega|} \sum_{n=2}^{\infty} \frac{1}{(n-1)^{\alpha}}<\infty
$$

one can deduce from the well-known Weierstrass test that the series

$$
\sum_{n=2}^{\infty} \Theta_{n}(t)
$$

is convergent in the sense of the norm $\|\cdot\|_{S^{p}}$ uniformly on $\mathbb{R}$. Now let

$$
\Delta(t):=\sum_{n=2}^{\infty} \Theta_{n}(t), \quad \text { for each } t \in \mathbb{R} .
$$

Observe that

$$
\Delta(t)=\int_{-\infty}^{t} S_{\alpha}(t-\sigma) G(\sigma) \mathrm{d} \sigma, \quad \text { for each } t \in \mathbb{R} .
$$

Clearly, for any $t, h \in \mathbb{R}$,

$$
\begin{aligned}
& \left(\int_{t}^{t+1}\|\Delta(\sigma+h)-\Delta(\sigma)\|^{p} \mathrm{~d} \sigma\right)^{\frac{1}{p}} \\
& \quad=\left(\int_{t}^{t+1}\left\|\int_{-\infty}^{\sigma+h} S_{\alpha}(\sigma+h-s) \Phi(s) \mathrm{d} s-\int_{-\infty}^{\sigma} S_{\alpha}(\sigma-s) \Phi(s) \mathrm{d} s\right\|^{p} \mathrm{~d} \sigma\right)^{\frac{1}{p}} \\
& \quad=\left(\int_{t}^{t+1}\left\|\int_{-\infty}^{\sigma} S_{\alpha}(\sigma-s)[\Phi(s+h)-\Phi(s)] \mathrm{d} s\right\|^{p} \mathrm{~d} \sigma\right)^{\frac{1}{p}} \\
& \quad=\left(\int_{t}^{t+1}\left\|\int_{0}^{\infty} S_{\alpha}(\tau)[\Phi(\sigma-\tau+h)-\Phi(\sigma-\tau)] \mathrm{d} \tau\right\|^{p} \mathrm{~d} \sigma\right)^{\frac{1}{p}} \\
& \quad \leq C M\|\Phi(t+h)-\Phi(t)\|_{S^{p}}\left(\int_{0}^{\infty}\left[\frac{1}{1+|\omega| \tau^{\alpha}}\right]^{p} \mathrm{~d} \tau\right)^{\frac{1}{p}} \\
& \quad \leq C M\|\Phi(t+h)-\Phi(t)\|_{S^{p}}\left(\int_{0}^{\infty} \frac{1}{1+|\omega| \tau^{\alpha}} \mathrm{d} \tau\right)^{\frac{1}{p}} \\
& \quad=C M\left[\frac{\omega^{-\frac{1}{\alpha}} \pi}{\alpha \sin \frac{\pi}{\alpha}}\right]^{\frac{1}{p}}\|\Phi(t+h)-\Phi(t)\|_{S^{p}},
\end{aligned}
$$

which shows that $\Delta$ is continuous. So, we only need to show that

$$
\lim _{T \rightarrow \infty} \frac{1}{m(T, \rho)} \int_{-T}^{T}\|\Delta(t)\| \rho(t) \mathrm{d} t=0
$$

In fact, one has

$$
\begin{aligned}
\left\|\Theta_{n}(t)\right\| & =\left\|\int_{t-n}^{t-n+1} S_{\alpha}(t-\sigma) \Phi(\sigma) \mathrm{d} \sigma\right\| \\
& \leq \int_{t-n}^{t-n+1}\left\|S_{\alpha}(t-\sigma)\right\|\|\Phi(\sigma)\| \mathrm{d} \sigma
\end{aligned}
$$




$$
\begin{aligned}
& \leq \int_{t-n}^{t-n+1} \frac{C M}{1+|\omega|(t-\sigma)^{\alpha}}\|\Phi(\sigma)\| \mathrm{d} \sigma \\
& \leq\left(\int_{t-n}^{t-n+1}\left[\frac{C M}{1+|\omega|(t-\sigma)^{\alpha}}\right]^{q}\right)^{\frac{1}{q}}\left(\int_{t-n}^{t-n+1}\|\Phi(\sigma)\|^{p} \mathrm{~d} \sigma\right)^{\frac{1}{p}} \\
& \leq \frac{C M}{1+|\omega|(n-1)^{\alpha}}\left(\int_{t-n}^{t-n+1}\|\Phi(\sigma)\|^{p} \mathrm{~d} \sigma\right)^{\frac{1}{p}} \\
& \leq \frac{C M}{|\omega|} \frac{1}{(n-1)^{\alpha}}\left(\int_{t-n}^{t-n+1}\|\Phi(\sigma)\|^{p} \mathrm{~d} \sigma\right)^{\frac{1}{p}},
\end{aligned}
$$

where $q=p /(p-1)$. Then

$$
\begin{aligned}
& \frac{1}{m(T, \rho)} \int_{-T}^{T}\left\|\Theta_{n}(t)\right\| \rho(t) \mathrm{d} t \\
& \quad \leq \frac{C M}{|\omega|} \frac{1}{(n-1)^{\alpha}} \frac{1}{m(T, \rho)} \int_{-T}^{T}\left(\int_{t-n}^{t-n+1}\|\Phi(\sigma)\|^{p} \mathrm{~d} \sigma\right)^{\frac{1}{p}} \rho(t) \mathrm{d} t
\end{aligned}
$$

and hence

$$
\Theta_{n}(t) \in P A A_{0}(\mathbb{R}, X, \rho)
$$

since

$$
\Phi \in P A A_{0}\left(\mathbb{R}, L^{p}([0,1], X), \rho\right) .
$$

From

$$
\Theta_{n}(t) \in P A A_{0}(\mathbb{R}, X, \rho)
$$

and

$$
\begin{aligned}
\frac{1}{m(T, \rho)} \int_{-T}^{T}\|\Psi(t)\| \rho(t) \mathrm{d} t \\
\leq \frac{C M}{|\omega|} \frac{1}{(n-1)^{\alpha}} \frac{1}{m(T, \rho)} \int_{-T}^{T}\left\|\Psi(t)-\sum_{n=2}^{N} \Theta_{n}(t)\right\| \rho(t) \mathrm{d} t \\
\quad+\sum_{n=2}^{N} \frac{C M}{|\omega|} \frac{1}{(n-1)^{\alpha}} \frac{1}{m(T, \rho)} \int_{-T}^{T}\left\|\Theta_{n}(t)\right\| \rho(t) \mathrm{d} t,
\end{aligned}
$$

it follows that

$$
\Psi(t) \in P A A_{0}(\mathbb{R}, X, \rho) .
$$

Therefore,

$$
x(t) \in S^{p} W P A A(\mathbb{R}, X) .
$$

The proof is now complete. 


\section{Stepanov-like almost automorphic mild solutions}

Let $1<\alpha<2$. We first consider the linear version for (1), that is

$$
D_{t}^{\alpha} x(t)=A x(t)+D_{t}^{\alpha-1} F(t), \quad t \in \mathbb{R} .
$$

Observe that (10) can be viewed as the limiting equation for the equation

$$
y^{\prime}(t)=\int_{0}^{t} \frac{(t-s)^{\alpha-2}}{\Gamma(\alpha-1)} A y(s)+F(t), \quad t \geq 0, y(0)=x_{0} \in X
$$

in the sense that the solutions $x(t)$ of (10) and $y(t)$ of (11) are asymptotic to each other as $t \rightarrow \infty$. In fact, if we assume that $A$ is sectorial of type $\omega$ with

$$
0 \leq \theta<\pi\left(1-\frac{\alpha}{2}\right)
$$

then (11) is well posed ( $c f .[88])$ and the variation of parameters formula allows us to write the solution of (11) as

$$
y(t)=S_{\alpha}(t) x_{0}+\int_{0}^{t} S_{\alpha}(t-s) F(s) \mathrm{d} s, \quad t \geq 0,
$$

where the family of operators $S_{\alpha}(t)$ is given by (5). On the other hand, if $S_{\alpha}(t)$ is integrable, then the solution of (10) is given by

$$
x(t)=\int_{-\infty}^{t} S_{\alpha}(t-s) F(s) \mathrm{d} s .
$$

Hence

$$
y(t)-x(t)=S_{\alpha}(t) x_{0}-\int_{t}^{\infty} S_{\alpha}(s) F(t-s) \mathrm{d} s,
$$

which shows that

$$
y(t)-x(t) \rightarrow 0, \quad \text { as } t \rightarrow \infty
$$

whenever $F \in L^{p}\left(\mathbb{R}^{+}, X\right)$ for some $p \in[1,+\infty)$.

From Cuesta's result, it follows that $S_{\alpha}(t)$ is integrable. Thus the above considerations motivate the following definition.

Definition 3.1 A function $x: \mathbb{R} \rightarrow X$ is said to be a mild solution to (10) if the function

$$
s \rightarrow S_{\alpha}(t-s) F(s)
$$

is integrable on $(-\infty, t)$ for each $t \in \mathbb{R}$ and

$$
x(t)=\int_{-\infty}^{t} S_{\alpha}(t-\sigma) F(\sigma) \mathrm{d} \sigma .
$$


Similarly, a function $x: \mathbb{R} \rightarrow X$ is said to be a mild solution to (1) if the function

$$
s \rightarrow S_{\alpha}(t-s) F(s, x(s))
$$

is integrable on $(-\infty, t)$ for each $t \in \mathbb{R}$ and

$$
x(t)=\int_{-\infty}^{t} S_{\alpha}(t-\sigma) F(\sigma, x(\sigma)) \mathrm{d} \sigma .
$$

To study the existence and uniqueness of weighted Stepanov-like pseudo-almost automorphic mild solutions to (1), we first consider the existence and uniqueness of weighted Stepanov-like pseudo-almost automorphic mild solutions to the linear fractional differential equation (10) with $1<\alpha<2$,

$$
A: D(A) \subset X \rightarrow X
$$

is a linear densely defined operator of sectorial type of $\omega<0$ on a complex Banach space $X$ and

$$
F: \mathbb{R} \rightarrow X
$$

is a weighted Stepanov-like pseudo-almost automorphic function. The fractional derivative is understood in the Riemann-Liouville sense.

The following are the main results for the linear fractional differential equations (10).

Theorem 3.1 Assume that $A$ is sectorial of type $\omega<0$. Then (10) admits a weighted Stepanov-like pseudo-almost automorphic mild solution.

Proof Since

$$
F \in S^{p} W P A A(\mathbb{R}, X),
$$

there exist

$$
G \in S^{p} A A(\mathbb{R}, X) \quad \text { and } \quad \Phi \in P A A_{0}\left(\mathbb{R}, L^{p}([0,1], X), \rho\right) \text {, }
$$

such that $F=G+\Phi$. So

$$
\begin{aligned}
x(t) & =\int_{-\infty}^{t} S_{\alpha}(t-\sigma) F(\sigma) \mathrm{d} \sigma \\
& =\int_{-\infty}^{t} S_{\alpha}(t-\sigma) G(\sigma) \mathrm{d} \sigma+\int_{-\infty}^{t} S_{\alpha}(t-\sigma) \Phi(\sigma) \mathrm{d} \sigma \\
& =\Xi_{1}(t)+\Xi_{2}(t) .
\end{aligned}
$$

We only need to verify

$$
\Xi_{1}(t) \in S^{p} A A(\mathbb{R}, X), \quad \Xi_{2}(t) \in P A A_{0}(\mathbb{R}, X, \rho) .
$$


First we prove that

$$
\Xi_{1}(t) \in S^{p} A A(\mathbb{R}, X) .
$$

Consider for each $n=1,2, \ldots$, the integrals

$$
\Upsilon_{n}(t)=\int_{t-n}^{t-n+1} S_{\alpha}(t-\sigma) G(\sigma) \mathrm{d} \sigma
$$

for each $t \in \mathbb{R}$. Note that

$$
\Upsilon_{n}(t)=\int_{t-n}^{t-n+1} S_{\alpha}(t-\sigma) G(\sigma) \mathrm{d} \sigma=\int_{n-1}^{n} S_{\alpha}(\sigma) G(t-\sigma) \mathrm{d} \sigma
$$

and by using the Hölder inequality, one gets

$$
\begin{aligned}
& \left\|\Upsilon_{n}(t)\right\|_{S^{p}}=\sup _{t \in \mathbb{R}}\left(\int_{t}^{t+1}\left\|\Upsilon_{n}(\tau)\right\|^{p} \mathrm{~d} \tau\right)^{\frac{1}{p}} \\
& =\sup _{t \in \mathbb{R}}\left(\int_{0}^{1}\left\|\Upsilon_{n}(t+s)\right\|^{p} \mathrm{~d} s\right)^{\frac{1}{p}} \\
& =\sup _{t \in \mathbb{R}}\left(\int_{0}^{1}\left\|\Upsilon_{n}(t+s)\right\|^{p} \mathrm{~d} s\right)^{\frac{1}{p}} \\
& =\sup _{t \in \mathbb{R}}\left(\int_{0}^{1}\left\|\int_{n-1}^{n} S_{\alpha}(\sigma) G(t+s-\sigma) \mathrm{d} \sigma\right\|^{p} \mathrm{~d} s\right)^{\frac{1}{p}} \\
& \leq \sup _{t \in \mathbb{R}}\left(\int_{0}^{1}\left(\int_{n-1}^{n}\left\|S_{\alpha}(\sigma)\right\|\|G(t+s-\sigma)\| \mathrm{d} \sigma\right)^{p} \mathrm{~d} s\right)^{\frac{1}{p}} \\
& \leq \sup _{t \in \mathbb{R}}\left(\int_{0}^{1} \int_{n-1}^{n}\left\|S_{\alpha}(\sigma)\right\|^{p}\|G(t+s-\sigma)\|^{p} \mathrm{~d} \sigma \mathrm{d} s\right)^{\frac{1}{p}} \\
& \leq \sup _{t \in \mathbb{R}}\left(\int_{0}^{1} \int_{n-1}^{n}\left[\frac{C M}{1+|\omega| \sigma^{\alpha}}\right]^{p}\|G(t+s-\sigma)\|^{p} \mathrm{~d} \sigma \mathrm{d} s\right)^{\frac{1}{p}} \\
& =\sup _{t \in \mathbb{R}}\left(\int_{n-1}^{n}\left[\frac{C M}{1+|\omega| \sigma^{\alpha}}\right]^{p} \int_{0}^{1}\|G(t+s-\sigma)\|^{p} \mathrm{~d} s \mathrm{~d} \sigma\right)^{\frac{1}{p}} \\
& =\sup _{t \in \mathbb{R}}\left(\int_{n-1}^{n}\left[\frac{C M}{1+|\omega| \sigma^{\alpha}}\right]^{p}\|G\|_{S^{p}}^{p} \mathrm{~d} \sigma\right)^{\frac{1}{p}} \\
& =C M\|G\|_{S^{p}}\left(\int_{n-1}^{n}\left[\frac{1}{1+|\omega| \sigma^{\alpha}}\right]^{p} \mathrm{~d} \sigma\right)^{\frac{1}{p}} \\
& \leq C M\|G\|_{S^{p}} \frac{1}{1+|\omega|(n-1)^{\alpha}} \\
& \leq \frac{C M\|G\|_{S^{p}}}{|\omega|} \frac{1}{(n-1)^{\alpha}} \text {. }
\end{aligned}
$$


From $1<\alpha<2$, it follows that

$$
\frac{C M\|G\|_{S^{p}}^{p}}{|\omega|} \sum_{n=2}^{\infty} \frac{1}{(n-1)^{\alpha}}<\infty
$$

one can deduce from the well-known Weierstrass test that the series

$$
\sum_{n=2}^{\infty} \Upsilon_{n}(t)
$$

is convergent in the sense of the norm $\|\cdot\|_{S^{p}}$ uniformly on $\mathbb{R}$. Now let

$$
\Phi(t):=\sum_{n=2}^{\infty} \Upsilon_{n}(t), \quad \text { for each } t \in \mathbb{R}
$$

Observe that

$$
\Phi(t)=\int_{-\infty}^{t} S_{\alpha}(t-\sigma) G(\sigma) \mathrm{d} \sigma, \quad \text { for each } t \in \mathbb{R}
$$

Clearly, for any $t, h \in \mathbb{R}$,

$$
\begin{aligned}
& \left(\int_{t}^{t+1}\|\Phi(\sigma+h)-\Phi(\sigma)\|^{p} \mathrm{~d} \sigma\right)^{\frac{1}{p}} \\
& \quad=\left(\int_{t}^{t+1}\left\|\int_{-\infty}^{\sigma+h} S_{\alpha}(\sigma+h-s) G(s) \mathrm{d} s-\int_{-\infty}^{\sigma} S_{\alpha}(\sigma-s) G(s) \mathrm{d} s\right\|^{p} \mathrm{~d} \sigma\right)^{\frac{1}{p}} \\
& =\left(\int_{t}^{t+1}\left\|\int_{-\infty}^{\sigma} S_{\alpha}(\sigma-s)[G(s+h)-G(s)] \mathrm{d} s\right\|^{p} \mathrm{~d} \sigma\right)^{\frac{1}{p}} \\
& \quad=\left(\int_{t}^{t+1}\left\|\int_{0}^{\infty} S_{\alpha}(\tau)[G(\sigma-\tau+h)-G(\sigma-\tau)] \mathrm{d} \tau\right\|^{p} \mathrm{~d} \sigma\right)^{\frac{1}{p}} \\
& \leq C M\|G(t+h)-G(t)\|_{S^{p}}\left(\int_{0}^{\infty}\left[\frac{1}{1+|\omega| \tau^{\alpha}}\right]^{p} \mathrm{~d} \tau\right)^{\frac{1}{p}} \\
& \leq C M\|G(t+h)-G(t)\|_{S^{p}}\left(\int_{0}^{\infty} \frac{1}{1+|\omega| \tau^{\alpha}} \mathrm{d} \tau\right)^{\frac{1}{p}} \\
& \quad=C M\left[\frac{\omega^{-\frac{1}{\alpha}} \pi}{\alpha \sin \frac{\pi}{\alpha}}\right]^{\frac{1}{p}}\|G(t+h)-G(t)\|_{S^{p}},
\end{aligned}
$$

which shows that $\Phi$ is continuous.

Now let us show that each

$$
\Upsilon_{n} \in S^{p} A A(\mathbb{R}, X)
$$

Indeed, let $\left\{s_{m}^{\prime}\right\}_{m \in \mathbb{N}}$ be a sequence of real numbers. Since

$$
G \in S^{p} A A(\mathbb{R}, X),
$$


there exist a subsequence $\left\{s_{m}\right\}_{m \in \mathbb{N}}$ of $\left\{s_{m}^{\prime}\right\}_{m \in \mathbb{N}}$ and a function $\tilde{G}$ such that

$$
\begin{aligned}
& {\left[\int_{0}^{1}\left\|G\left(t+s_{n}+s\right)-\tilde{G}(t+s)\right\|^{p} \mathrm{~d} s\right]^{\frac{1}{p}} \rightarrow 0,} \\
& {\left[\int_{0}^{1}\left\|\tilde{G}\left(t-s_{n}+s\right)-G(t+s)\right\|^{p} \mathrm{~d} s\right]^{\frac{1}{p}} \rightarrow 0,}
\end{aligned}
$$

as $m \rightarrow \infty$ pointwise on $\mathbb{R}$. Moreover, if we let

$$
\tilde{\Upsilon}_{n}(t)=\int_{n-1}^{n} S_{\alpha}(\sigma) \tilde{G}(t-\sigma) \mathrm{d} \sigma
$$

one has

$$
\begin{aligned}
& \left(\int_{0}^{1}\left\|x_{n}\left(t+s+s_{m}\right)-\tilde{x}_{n}(t+s)\right\|^{p} \mathrm{~d} s\right)^{\frac{1}{p}} \\
& \quad=\left(\int_{0}^{1}\left\|\int_{n-1}^{n} S_{\alpha}(\sigma) G\left(t+s+s_{m}-\sigma\right) \mathrm{d} \sigma-\int_{n-1}^{n} S_{\alpha}(\sigma) \tilde{G}(t+s-\sigma) \mathrm{d} \sigma\right\|^{p} \mathrm{~d} s\right)^{\frac{1}{p}} \\
& \quad=\left(\int_{0}^{1}\left\|\int_{n-1}^{n} S_{\alpha}(\sigma)\left[G\left(t+s+s_{m}-\sigma\right)-\tilde{G}(t+s-\sigma)\right] \mathrm{d} \sigma\right\|^{p} \mathrm{~d} s\right)^{\frac{1}{p}} \\
& \quad \leq\left(\int_{0}^{1}\left(\int_{n-1}^{n}\left\|S_{\alpha}(\sigma)\right\|\left\|G\left(t+s+s_{m}-\sigma\right)-\tilde{G}(t+s-\sigma)\right\| \mathrm{d} \sigma\right)^{p} \mathrm{~d} s\right)^{\frac{1}{p}} \\
& \quad \leq\left(\int_{0}^{1} \int_{n-1}^{n}\left\|S_{\alpha}(\sigma)\right\|^{p}\left\|G\left(t+s+s_{m}-\sigma\right)-\tilde{G}(t+s-\sigma)\right\|^{p} \mathrm{~d} \sigma \mathrm{d} s\right)^{\frac{1}{p}} \\
& \quad \leq\left(\int_{0}^{1} \int_{n-1}^{n}\left[\frac{C M}{1+|\omega| \sigma^{\alpha}}\right]^{p}\left\|G\left(t+s+s_{m}-\sigma\right)-\tilde{G}(t+s-\sigma)\right\|^{p} \mathrm{~d} \sigma \mathrm{d} s\right)^{\frac{1}{p}} \\
& \quad=\left(\int_{n-1}^{n}\left[\frac{C M}{1+|\omega| \sigma^{\alpha}}\right]^{p} \int_{0}^{1}\left\|G\left(t+s+s_{m}-\sigma\right)-\tilde{G}(t+s-\sigma)\right\|^{p} \mathrm{~d} s \mathrm{~d} \sigma\right)^{\frac{1}{p}} .
\end{aligned}
$$

Obviously, the last inequality goes to 0 as $m \rightarrow \infty$ pointwise on $\mathbb{R}$. Similarly one can prove that

$$
\left[\int_{0}^{1}\left\|\tilde{\Upsilon}_{n}\left(t+s-s_{m}\right)-\Upsilon_{n}(t+s)\right\|^{p} \mathrm{~d} s\right]^{\frac{1}{p}} \rightarrow 0
$$

as $m \rightarrow \infty$ pointwise on $\mathbb{R}$. Thus we conclude that each

$$
\Upsilon_{n} \in S^{p} A A(\mathbb{R}, X)
$$

and consequently their uniform limit

$$
\Phi(t) \in S^{p} A A(\mathbb{R}, X)
$$

by using Lemma 2.7 . 
In the following, we prove that

$$
\Xi_{2}(t) \in P A A_{0}(\mathbb{R}, X, \rho) .
$$

To complete the proof, consider for each $n=1,2, \ldots$, the integrals

$$
\Theta_{n}(t)=\int_{t-n}^{t-n+1} S_{\alpha}(t-\sigma) \Phi(\sigma) \mathrm{d} \sigma
$$

for each $t \in \mathbb{R}$. Note that

$$
\Theta_{n}(t)=\int_{t-n}^{t-n+1} S_{\alpha}(t-\sigma) \Phi(\sigma) \mathrm{d} \sigma=\int_{n-1}^{n} S_{\alpha}(\sigma) \Phi(t-\sigma) \mathrm{d} \sigma .
$$

By carrying out similar arguments as above, we know that $\Theta_{n}(t)$ is bounded and continuous, and

$$
\sum_{n=2}^{\infty} \Theta_{n}(t)
$$

is uniformly convergent on $\mathbb{R}$. Let

$$
\Psi(t):=\sum_{n=2}^{\infty} \Theta_{n}(t), \quad \text { for each } t \in \mathbb{R}
$$

then

$$
\Psi(t)=\int_{-\infty}^{t} S_{\alpha}(t-\sigma) \Phi(\sigma) \mathrm{d} \sigma, \quad \text { for each } t \in \mathbb{R} .
$$

It is obvious that $\Psi(t)$ is bounded and continuous. So, we only need to show that

$$
\lim _{T \rightarrow \infty} \frac{1}{m(T, \rho)} \int_{-T}^{T}\|\Psi(t)\| \rho(t) \mathrm{d} t=0 .
$$

In fact, one has

$$
\begin{aligned}
\left\|\Theta_{n}(t)\right\| & =\left\|\int_{t-n}^{t-n+1} S_{\alpha}(t-\sigma) \Phi(\sigma) \mathrm{d} \sigma\right\| \\
& \leq \int_{t-n}^{t-n+1}\left\|S_{\alpha}(t-\sigma)\right\|\|\Phi(\sigma)\| \mathrm{d} \sigma \\
& \leq \int_{t-n}^{t-n+1} \frac{C M}{1+|\omega|(t-\sigma)^{\alpha}}\|\Phi(\sigma)\| \mathrm{d} \sigma \\
& \leq\left(\int_{t-n}^{t-n+1}\left[\frac{C M}{1+|\omega|(t-\sigma)^{\alpha}}\right]^{q}\right)^{\frac{1}{q}}\left(\int_{t-n}^{t-n+1}\|\Phi(\sigma)\|^{p} \mathrm{~d} \sigma\right)^{\frac{1}{p}} \\
& \leq \frac{C M}{1+|\omega|(n-1)^{\alpha}}\left(\int_{t-n}^{t-n+1}\|\Phi(\sigma)\|^{p} \mathrm{~d} \sigma\right)^{\frac{1}{p}} \\
& \leq \frac{C M}{|\omega|} \frac{1}{(n-1)^{\alpha}}\left(\int_{t-n}^{t-n+1}\|\Phi(\sigma)\|^{p} \mathrm{~d} \sigma\right)^{\frac{1}{p}}
\end{aligned}
$$


where $q=p /(p-1)$. Then

$$
\begin{aligned}
& \frac{1}{m(T, \rho)} \int_{-T}^{T}\left\|\Theta_{n}(t)\right\| \rho(t) \mathrm{d} t \\
& \quad \leq \frac{C M}{|\omega|} \frac{1}{(n-1)^{\alpha}} \frac{1}{m(T, \rho)} \int_{-T}^{T}\left(\int_{t-n}^{t-n+1}\|\Phi(\sigma)\|^{p} \mathrm{~d} \sigma\right)^{\frac{1}{p}} \rho(t) \mathrm{d} t
\end{aligned}
$$

and hence

$$
\Theta_{n}(t) \in P A A_{0}(\mathbb{R}, X, \rho)
$$

since

$$
\Phi \in P A A_{0}\left(\mathbb{R}, L^{p}([0,1], X), \rho\right) .
$$

From

$$
\Theta_{n}(t) \in P A A_{0}(\mathbb{R}, X, \rho)
$$

and

$$
\begin{aligned}
\frac{1}{m(T, \rho)} \int_{-T}^{T}\|\Psi(t)\| \rho(t) \mathrm{d} t \\
\leq \frac{C M}{|\omega|} \frac{1}{(n-1)^{\alpha}} \frac{1}{m(T, \rho)} \int_{-T}^{T}\left\|\Psi(t)-\sum_{n=2}^{N} \Theta_{n}(t)\right\| \rho(t) \mathrm{d} t \\
\quad+\sum_{n=2}^{N} \frac{C M}{|\omega|} \frac{1}{(n-1)^{\alpha}} \frac{1}{m(T, \rho)} \int_{-T}^{T}\left\|\Theta_{n}(t)\right\| \rho(t) \mathrm{d} t,
\end{aligned}
$$

it follows that

$$
\Psi(t) \in P A A_{0}(\mathbb{R}, X, \rho) .
$$

Therefore,

$$
x(t) \in S^{p} W P A A(\mathbb{R}, X) .
$$

In view of the above, it follows that $x(t)$ is the bounded weighted Stepanov-like pseudoalmost automorphic mild solution to (10). The proof is now complete.

Now we investigate the Stepanov-like almost automorphic mild solutions to the nonlinear fractional differential equation (1), the following are the main results.

Theorem 3.2 Assume that $A$ is sectorial of type $\omega<0$ and $\rho \in U_{\infty}$. Let

$$
F=G+\Phi \in S^{p} W P A A(\mathbb{R} \times X, X, \rho)
$$


with

$$
G^{b} \in A A\left(\mathbb{R} \times X, L^{p}([0,1], X)\right), \quad \Phi \in P A A_{0}\left(\mathbb{R} \times X, L^{p}([0,1], X)\right),
$$

and there exist nonnegative functions

$$
L_{F}, L_{G} \in S^{r} A A(\mathbb{R}, \mathbb{R})
$$

with

$$
r \geq \max \left\{p, \frac{p}{p-1}\right\}
$$

such that

$$
\begin{aligned}
& \|F(t, x)-F(t, y)\| \leq L_{F}(t)\|x-y\|, \\
& \|G(t, x)-G(t, y)\| \leq L_{G}(t)\|x-y\|, \quad x, y \in X, t \in \mathbb{R},
\end{aligned}
$$

where

$$
L_{F}(t) \in L^{p}(\mathbb{R}) .
$$

Then (1) admits a unique weighed Stepanov-like pseudo-almost automorphic mild solution.

Proof Define the operator $\Gamma$ on $S^{p} W P A A(\mathbb{R}, X)$ by

$$
\Gamma x(t)=\int_{-\infty}^{t} S_{\alpha}(t-\sigma) F(\sigma, x(\sigma)) \mathrm{d} \sigma .
$$

From Lemma 2.6, it follows that

$$
F(\cdot)=F(\cdot, x(\cdot)) \in S^{p} W P A A(\mathbb{R}, X) .
$$

From the function

$$
\frac{1}{1+|\omega| t^{\alpha}}
$$

being integrable on $\mathbb{R}^{+}(\alpha>1)$ and the proof of Lemma 2.7 , one can easily see that $\Gamma x$ is well defined and continuous. Then by using the proof of Theorem 3.1 with the above Lemma 2.8, one has

$$
\Gamma x \in S^{p} W P A A(\mathbb{R}, X)
$$

whenever

$$
x \in S^{p} W P A A(\mathbb{R}, X) .
$$


Thus $\Gamma$ maps $S^{p} W P A A(\mathbb{R}, X)$ into itself. It suffices now to show that this operator $\Gamma$ has a unique fixed point in $S^{p} W P A A(\mathbb{R}, X)$. For this, let $x, y$ be in $S^{p} W P A A(\mathbb{R}, X)$ and define

$$
C_{\alpha}:=\sup _{t \in \mathbb{R}}\left\|S_{\alpha}(t)\right\|
$$

one has

$$
\begin{aligned}
& \|\Gamma x(t)-\Gamma y(t)\|_{S^{p}} \\
& \quad=\sup _{t \in \mathbb{R}}\left(\int_{t}^{t+1}\left\|\int_{-\infty}^{\tau} S_{\alpha}(\tau-\sigma)[F(\sigma, x(\sigma))-F(\sigma, y(\sigma))] \mathrm{d} \sigma\right\|^{p} \mathrm{~d} \tau\right)^{\frac{1}{p}} \\
& \quad \leq \sup _{t \in \mathbb{R}}\left(\int_{t}^{t+1} \int_{-\infty}^{\tau} L^{p}(\sigma)\|S(\tau-\sigma)\|^{p}\|x(\sigma)-y(\sigma)\|^{p} \mathrm{~d} \sigma \mathrm{d} \tau\right)^{\frac{1}{p}} \\
& \quad \leq C_{\alpha}\|L\|_{p}\|x-y\|_{S^{p}} .
\end{aligned}
$$

In general we get

$$
\begin{aligned}
& \left\|\left[\Gamma^{n} x\right](t)-\left[\Gamma^{n} y\right](t)\right\|_{S^{p}} \\
& \quad \leq \frac{C_{\alpha}^{n}}{(n-1) !}\left(\int_{-\infty}^{t} L^{p}(\sigma)\left(\int_{-\infty}^{\sigma} L^{p}(\tau) \mathrm{d} \tau\right)^{n-1} \mathrm{~d} \sigma\right)^{\frac{1}{p}}\|x-y\|_{S^{p}} \\
& \quad \leq \frac{C_{\alpha}^{n}}{n !}\left(\left(\int_{-\infty}^{t} L(\sigma) \mathrm{d} \sigma\right)^{\frac{1}{p}}\right)^{n}\|x-y\|_{S^{p}} \\
& \quad \leq \frac{\left(C_{\alpha}\|L\|_{p}\right)^{n}}{n !}\|x-y\|_{S^{p}} .
\end{aligned}
$$

Hence, since

$$
\frac{\left(C_{\alpha}\|L\|_{p}\right)^{n}}{n !}<1
$$

for $n$ sufficiently large, by the contraction principle $\Gamma$ has a unique fixed point

$$
x \in S^{p} W P A A(\mathbb{R}, X) .
$$

We note that conditions of type (13) have been previously considered in the literature for almost automorphic functions [90]. Our motivation comes from their use in the study of pseudo-almost periodic solutions of semilinear Cauchy problems [91]. Now we consider the more general case of equations introducing a new class of functions $L$ which do not necessarily belong to $L^{p}(\mathbb{R})$. We have the following result.

Theorem 3.3 Assume that $A$ is sectorial of type $\omega<0$ and $\rho \in U_{\infty}$. Let

$$
F=G+\Phi \in S^{p} W P A A(\mathbb{R} \times X, X, \rho)
$$

with

$$
G^{b} \in A A\left(\mathbb{R} \times X, L^{p}([0,1], X)\right), \quad \Phi \in P A A_{0}\left(\mathbb{R} \times X, L^{p}([0,1], X)\right),
$$


and there exist nonnegative functions

$$
L_{F}, L_{G} \in S^{r} A A(\mathbb{R}, \mathbb{R})
$$

with

$$
r \geq \max \left\{p, \frac{p}{p-1}\right\}
$$

such that

$$
\begin{aligned}
& \|F(t, x)-F(t, y)\| \leq L_{F}(t)\|x-y\|, \\
& \|G(t, x)-G(t, y)\| \leq L_{G}(t)\|x-y\|, \quad x, y \in X, t \in \mathbb{R},
\end{aligned}
$$

where the integral

$$
\int_{-\infty}^{t} L_{F}(\sigma) \mathrm{d} \sigma
$$

exists for all $t \in \mathbb{R}$. Then (1) admits a unique weighed Stepanov-like pseudo-almost automorphic mild solution.

Proof Define a new norm

$$
|\|x\||:=\sup _{t \in \mathbb{R}}\left\{v(t)\|x(t)\|_{S^{p}}\right\},
$$

where

$$
v(t):=\left[e^{-k \int_{-\infty}^{t} L(\sigma) \mathrm{d} \sigma}\right]^{\frac{1}{p}},
$$

and $k$ is a fixed positive constant greater than

$$
C_{\alpha}:=\sup _{t \in \mathbb{R}}\left\|S_{\alpha}(t)\right\|
$$

Let $x, y$ be in $S^{p} W P A A(\mathbb{R}, X)$, then one has

$$
\begin{aligned}
& v(t)\|\Gamma x(t)-\Gamma y(t)\|_{S^{p}} \\
& =v(t) \sup _{t \in \mathbb{R}}\left(\int_{t}^{t+1}\left\|\int_{-\infty}^{\tau} S(\tau-\sigma)[F(\sigma, x(\sigma))-F(\sigma, y(\sigma))] \mathrm{d} \sigma\right\|^{p} \mathrm{~d} \tau\right)^{\frac{1}{p}} \\
& \quad \leq C_{\alpha} \sup _{t \in \mathbb{R}}\left(\int_{t}^{t+1} \int_{-\infty}^{\tau} v^{p}(\tau) L^{p}(\sigma)\|x(\sigma)-y(\sigma)\|^{p} \mathrm{~d} \sigma \mathrm{d} \tau\right)^{\frac{1}{p}} \\
& =C_{\alpha} \sup _{t \in \mathbb{R}}\left(\int_{t}^{t+1} \int_{-\infty}^{\tau} v^{p}(\tau) v^{p}(\sigma) L^{p}(\sigma)\left(v^{p}(\sigma)\right)^{-1}\|x(\sigma)-y(\sigma)\|^{p} \mathrm{~d} \sigma \mathrm{d} \tau\right)^{\frac{1}{p}} \\
& \quad \leq C_{\alpha}|\|x-y\|| \sup _{t \in \mathbb{R}}\left(\int_{t}^{t+1} \int_{-\infty}^{\tau} v^{p}(\tau)\left(v^{p}(\sigma)\right)^{-1} L^{p}(\sigma) \mathrm{d} \sigma \mathrm{d} \tau\right)^{\frac{1}{p}}
\end{aligned}
$$




$$
\begin{aligned}
& =\frac{C_{\alpha}}{k}|\|x-y\|| \sup _{t \in \mathbb{R}}\left(\int_{t}^{t+1} \int_{-\infty}^{\tau} k e^{k \int_{\tau}^{\sigma} L(\tau) \mathrm{d} \tau} L(\sigma) \mathrm{d} \sigma \mathrm{d} \tau\right)^{\frac{1}{p}} \\
& =\frac{C_{\alpha}}{k}|\|x-y\|| \sup _{t \in \mathbb{R}}\left(\int_{t}^{t+1} \int_{-\infty}^{\tau} \frac{\mathrm{d}}{\mathrm{d} \sigma}\left(e^{k \int_{\tau}^{\sigma} L(\tau) \mathrm{d} \tau}\right) \mathrm{d} \sigma \mathrm{d} \tau\right)^{\frac{1}{p}} \\
& =\frac{C_{\alpha}}{k} \sup _{t \in \mathbb{R}}\left(\int_{t}^{t+1}\left(1-e^{-k \int_{-\infty}^{\tau} L(\tau) \mathrm{d} \tau}\right) \mathrm{d} \tau\right)^{\frac{1}{p}}|\|x-y\|| \\
& \leq \frac{C_{\alpha}}{k}|\|x-y\|| .
\end{aligned}
$$

Hence, since

$$
\frac{C_{\alpha}}{k}<1
$$

$\Gamma$ has a unique fixed point

$$
x \in S^{p} W P A A(\mathbb{R}, X) .
$$

Note that the above result does not include the cases where $L_{F}$ and $L_{G}$ are constants.

Theorem 3.4 Assume that $A$ is sectorial of type $\omega<0$ and $\rho \in U_{\infty}$. Let

$$
F=G+\Phi \in S^{p} W P A A(\mathbb{R} \times X, X, \rho)
$$

with

$$
G^{b} \in A A\left(\mathbb{R} \times X, L^{p}([0,1], X)\right), \quad \Phi \in P A A_{0}\left(\mathbb{R} \times X, L^{p}([0,1], X)\right),
$$

and there exist constants $L_{F}, L_{G}$ such that

$$
\begin{aligned}
& \|F(t, x)-F(t, y)\| \leq L_{F}\|x-y\|, \\
& \|G(t, x)-G(t, y)\| \leq L_{G}\|x-y\|, \quad x, y \in X, t \in \mathbb{R},
\end{aligned}
$$

Then (1) admits a unique Stepanov-like pseudo-almost automorphic mild solution whenever

$$
C M L_{F} \omega^{-\frac{1}{\alpha}} \pi<\alpha \sin \frac{\pi}{\alpha}
$$

Proof For $x, y \in S^{p} W P A A(\mathbb{R}, X)$, one has

$$
\begin{aligned}
& \|\Gamma x(t)-\Gamma y(t)\|_{S^{p}} \\
& \quad=\sup _{t \in \mathbb{R}}\left(\int_{t}^{t+1}\left\|\int_{-\infty}^{s} S_{\alpha}(s-\sigma)[F(\sigma, x(\sigma))-F(\sigma, y(\sigma))] \mathrm{d} \sigma\right\|^{p} \mathrm{~d} s\right)^{\frac{1}{p}} \\
& \quad=\sup _{t \in \mathbb{R}}\left(\int_{t}^{t+1}\left\|\int_{0}^{\infty} S_{\alpha}(\tau)[F(s-\tau, x(s-\tau))-F(s-\tau, y(s-\tau))] \mathrm{d} \tau\right\|^{p} \mathrm{~d} s\right)^{\frac{1}{p}}
\end{aligned}
$$




$$
\begin{aligned}
& \leq L \sup _{t \in \mathbb{R}}\left(\int_{t}^{t+1} \int_{0}^{\infty}\left\|S_{\alpha}(\tau)\right\|^{p}\|x(s-\tau)-y(s-\tau)\|^{p} \mathrm{~d} \tau \mathrm{d} s\right)^{\frac{1}{p}} \\
& \leq C M L\|x-y\|_{S^{p}}\left(\int_{0}^{\infty}\left(\frac{1}{1+|\omega| \tau^{\alpha}}\right)^{p} \mathrm{~d} \tau \mathrm{d} s\right)^{\frac{1}{p}} \\
& \leq C M L\|x-y\|_{S^{p}}\left(\int_{0}^{\infty} \frac{1}{1+|\omega| \tau^{\alpha}} \mathrm{d} \tau \mathrm{d} s\right)^{\frac{1}{p}} \\
& =C M L\left[\frac{\omega^{-\frac{1}{\alpha}} \pi}{\alpha \sin \frac{\pi}{\alpha}}\right]^{\frac{1}{p}}\|x-y\|_{S^{p}} .
\end{aligned}
$$

This proves that $\Gamma$ is a strict contraction, so it follows from the Banach contraction mapping principle that $\Gamma$ admits a unique fixed point

$$
x \in S^{p} \operatorname{WPA} A(\mathbb{R}, X),
$$

which is the unique weighed Stepanov-like pseudo-almost automorphic mild solution to (1).

\section{Taking}

$$
A=-\rho^{\alpha} I, \quad \text { with } \rho>0
$$

in (1), the above theorem gives the following corollary.

Corollary 3.1 Let $\rho \in U_{\infty}$,

$$
F=G+\Phi \in S^{p} W P A A(\mathbb{R} \times X, X, \rho)
$$

with

$$
G^{b} \in A A\left(\mathbb{R} \times X, L^{p}([0,1], X)\right), \quad \Phi \in P A A_{0}\left(\mathbb{R} \times X, L^{p}([0,1], X)\right),
$$

and there exist constants $L_{F}, L_{G}$ such that

$$
\begin{aligned}
& \|F(t, x)-F(t, y)\| \leq L_{F}\|x-y\|, \\
& \|G(t, x)-G(t, y)\| \leq L_{G}\|x-y\|, \quad x, y \in X, t \in \mathbb{R} .
\end{aligned}
$$

Then (1) admits a unique weighted Stepanov-like pseudo-almost automorphic mild solution whenever

$$
C L_{F}<\frac{\alpha \sin \left(\frac{\pi}{\alpha}\right)}{\rho \pi} .
$$

Remark 3.1 It is interesting to note that the function

$$
\alpha \rightarrow \frac{\alpha \sin \left(\frac{\pi}{\alpha}\right)}{\rho \pi}
$$


is increasing from 0 to $\frac{2}{\rho \pi}$ in the interval $1<\alpha<2$. Therefore, with respect to the Lipschitz condition (15), the class of admissible semilinear terms $F(t, x(t))$ is the best in the case $\alpha=2$ and the worst in the case $\alpha=1$. Note the direct relation with the term

$$
\frac{(t-s)^{\alpha-2}}{\Gamma(\alpha-1)}
$$

in (11), where the singularity becomes better (smooth) when $\alpha$ goes from 1 to 2 .

\section{Applications}

In this section we give an example to illustrate the above results.

Consider the following fractional relaxation-oscillation equation:

$$
\begin{aligned}
& \partial_{t}^{\alpha} u(t, x)=\partial_{x}^{2} u(t, x)-\mu u(t, x)+\partial_{t}^{\alpha-1} F(t, u(t, x)), \quad t \in \mathbb{R}, x \in[0, \pi], \\
& u(t, 0)=u(t, \pi)=0, \quad t \in \mathbb{R},
\end{aligned}
$$

where $\mu>0, F: \mathbb{R} \times \mathbb{R} \rightarrow \mathbb{R}$ is a given function.

Take $X=L^{2}([0, \pi])$ and define the operator $A$ by

$$
A \varphi:=\varphi^{\prime \prime}-\mu \varphi, \quad \varphi \in D(A)
$$

where

$$
D(A):=\left\{\varphi \in L^{2}[0, \pi]: \varphi^{\prime \prime} \in L^{2}[0, \pi], \varphi(0)=\varphi(\pi)\right\} \subset L^{2}[0, \pi] .
$$

It is well known that

$$
A u=u^{\prime \prime}
$$

is the generator of an analytic semigroup on $L^{2}[0, \pi]$. Hence,

$$
\mu I-A
$$

is sectorial of type

$$
\omega=-\mu<0 .
$$

Equation (17) can be formulated by the inhomogeneous problem (1), where

$$
u(t)=u(t, \cdot) .
$$

Example 4.1 Let us consider the nonlinearity

$$
F(t, x)(s)=\beta e^{-t}\left[\sin \left(\frac{1}{2+\cos t+\cos \sqrt{2} t}\right) \sin (x(s))+\max \left\{e^{-\left(t \pm k^{2}\right)^{2}}\right\}\right],
$$

for all $x \in X$ and $s \in[0, \pi], t \in \mathbb{R}$. Thus one has

$$
F(t, x) \in S^{p} W P A A(\mathbb{R} \times X, X)
$$


and

$$
\|F(t, x)-F(t, y)\|_{2}^{2} \leq \int_{0}^{\pi} \beta^{2} e^{-2 t}|\sin (x(s))-\sin (y(s))| \mathrm{d} s \leq \beta^{2} e^{-2 t}\|x(s)-y(s)\|_{2}^{2} .
$$

In consequence, from Theorem 3.2, it follows that the fractional differential equation (17) has a unique weighed Stepanov-like pseudo-almost automorphic mild solution.

Example 4.2 Let us consider the nonlinearity

$$
F(t, x)(s)=\beta e^{-|t|}\left[\sin \left(\frac{1}{2+\cos t+\cos \sqrt{2} t}\right) \sin (x(s))+\max \left\{e^{-\left(t \pm k^{2}\right)^{2}}\right\}\right]
$$

for all $x \in X$ and $s \in[0, \pi], t \in \mathbb{R}$. Thus one has

$$
F(t, x) \in S^{p} W P A A(\mathbb{R} \times X, X)
$$

and

$$
\|F(t, x)-F(t, y)\|_{2}^{2} \leq \int_{0}^{\pi} \beta^{2} e^{-2|t|}|\sin (x(s))-\sin (y(s))| \mathrm{d} s \leq \beta^{2} e^{-2|t|}\|x(s)-y(s)\|_{2}^{2} .
$$

In consequence, from Theorem 3.3, it follows that the fractional differential equation (17) has a unique weighed Stepanov-like pseudo-almost automorphic mild solution.

Example 4.3 Let us consider the nonlinearity

$$
F(t, x)(s)=\beta e^{-\frac{t^{2}}{4}}\left[\sin \left(\frac{1}{2+\cos t+\cos \sqrt{2} t}\right) \sin (x(s))+\max \left\{e^{-\left(t \pm k^{2}\right)^{2}}\right\}\right],
$$

for all $x \in X$ and $s \in[0, \pi], t \in \mathbb{R}$. Thus one has

$$
F(t, x) \in S^{p} W P A A(\mathbb{R} \times X, X)
$$

and

$$
\|F(t, x)-F(t, y)\|_{2}^{2} \leq \int_{0}^{\pi} \beta^{2} e^{-\frac{t^{2}}{2}}|\sin (x(s))-\sin (y(s))| \mathrm{d} s \leq \beta^{2} e^{-\frac{t^{2}}{2}}\|x(s)-y(s)\|_{2}^{2} .
$$

In consequence, from Theorem 3.4 it follows that the fractional differential equation (17) has a unique weighed Stepanov-like pseudo-almost automorphic mild solution whenever

$$
|\beta|<\frac{\alpha \sin \left(\frac{\pi}{\alpha}\right)}{\pi C M|\mu|^{-\frac{1}{\alpha}}} .
$$




\section{Acknowledgements}

This work is supported by the National Natural Science Foundation of China (No. 11301090), Appropriative Researching Fund for Professors and Doctors, Guangdong University of Education (No. 2013ARF02).

Received: 28 October 2014 Accepted: 9 February 2015 Published online: 04 March 2015

\section{References}

1. Bochner, S: Beiträe zur theorie der fastperiodischen funktionen. Math. Ann. 96, 119-147 (1927)

2. Corduneanu, C: Almost Periodic Oscillations and Waves. Springer, New York (2009)

3. Kundert, K, Sorkin, G, Sangiovanni-Vincentelli, A: Applying harmonic balance to almost-periodic circuits. IEEE Trans. Microw. Theory Tech. 36, 366-378 (1988)

4. Ahmad, S: On almost periodic solutions of the competing species problems. Proc. Am. Math. Soc. 102, 855-861 (1988)

5. Fink, A: Almost Periodic Differential Equations. Lecture Notes in Math., vol. 37. Springer, Berlin (1974)

6. Hewitt, E: Linear functionals on almost periodic functions. Trans. Am. Math. Soc. 74, 303-322 (1953)

7. Doss, R: On generalized almost periodic functions. II. J. Lond. Math. Soc. 37, 133-140 (1962)

8. Batty, C, Hutter, W, Räiger, F: Almost periodicity of mild solutions of inhomogeneous periodic Cauchy problems. J. Differ. Equ. 156, 309-327 (1999)

9. Ortega, R, Tarallo, M: Almost periodic linear differential equations with non-separated solutions. J. Funct. Anal. 237, 402-426 (2006)

10. Huang, W, Yi, Y: Almost periodically forced circle flows. J. Funct. Anal. 257, 832-902 (2009)

11. Arendt, W, Batty, C: Almost periodic solutions of first and second order Cauchy problems. J. Differ. Equ. 137, 363-383 (1997)

12. Levitan, B, Zhikov, V: Almost Periodic Functions and Differential Equations. Cambridge University Press, Cambridge (1982)

13. Zhang, C: Almost Periodic Type Functions and Ergodicity. Science Press, Beijing (2003)

14. Agarwal, R, Cuevas, C, Soto, H, El-Gebeily, M: Asymptotic periodicity for some evolution equations in Banach spaces. Nonlinear Anal. 74, 1769-1798 (2011)

15. Cuevas, C, Sepúlveda, A, Soto, H: Almost periodic and pseudo-almost periodic solutions to fractional differential and integro-differential equations. Appl. Math. Comput. 218, 1735-1745 (2011)

16. Fréchet, M: Les fonctions asymptotiquement presque-périodiques. Revue Sci. (Rev. Rose Illus.) 79, 341 -354 (1941)

17. Zhang, C: Pseudo almost periodic functions and their applications. Thesis, The University of Western, Ontario (1992)

18. Diagana, T: Weighted pseudo almost periodic functions and applications. C. R. Math. Acad. Sci. Paris 343, 643-646 (2006)

19. Stepanov, W: Sur quelques généralizations des fonctions presque-périodiques. C. R. Acad. Sci. Paris 181, 90-94 (1925)

20. Stepanov, W: Über einige verallgemeinerungen der fastperiodischen funktionen. Math. Ann. 90, 473-492 (1925)

21. Diagana, T: Stepanov-like pseudo almost periodic functions and their applications to differential equations. Commun. Math. Anal. 3, 9-18 (2007)

22. Diagana, T, Mophou, G, N'Guérékata, G: Existence of weighted pseudo-almost periodic solutions to some classes of differential equations with $S^{P}$-weighted pseudo-almost periodic coefficients. Nonlinear Anal. 72, 430-438 (2010)

23. Bochner, S: Curvature and Betti numbers in real and complex vector bundles. Rend. Semin. Mat. (Torino) 15, 225-253 (1955)

24. Bochner, S: Uniform convergence of monotone sequences of functions. Proc. Natl. Acad. Sci. USA 47, $582-585$ (1961)

25. Bochner, S: A new approach to almost periodicity. Proc. Natl. Acad. Sci. USA 48, 2039-2043 (1962)

26. Bochner, S: Continuous mappings of almost automorphic and almost periodic functions. Proc. Natl. Acad. Sci. USA 52, 907-910 (1964)

27. N'Guérékata, G: Sur les solutions presque automorphes d'équations différentielles abstraites. Ann. Sci. Math. Qué. 1, 69-79 (1981)

28. Xiao, T, Liang, J, Zhang, J: Pseudo almost automorphic solutions to semilinear differential equations in Banach space. Semigroup Forum 76, 518-524 (2008)

29. Blot, J, Mophou, G, N'Guérékata, G, Pennequin, D: Weighted pseudo almost automorphic functions and applications to abstract differential equations. Nonlinear Anal., Theory Methods Appl. 71, 903-909 (2009)

30. Casarino, V: Characterization of almost automorphic groups and semigroups. Rend. Accad. Naz. Sci. XL Mem. Mat. Appl. (5) 24, 219-235 (2000)

31. Diagana, T: Existence of pseudo-almost automorphic solutions to some abstract differential equations with $S^{p}$-pseudo-almost automorphic coefficients. Nonlinear Anal., Theory Methods Appl. 70, 3781-3790 (2009)

32. Xia, Z, Fan, M: Weighted Stepanov-like pseudo almost automorphy and applications. Nonlinear Anal., Theory Methods Appl. 75, 2378-2397 (2012)

33. Diagana, T, N'Guérékata, G: Stepanov-like almost automorphic functions and applications to some semilinear equations. Appl. Anal. 86, 723-733 (2007)

34. Liu, J, N'Guérékata, G, Van Minh, N: A Massera type theorem for almost automorphic solutions of differential equations. J. Math. Anal. Appl. 299, 587-599 (2004)

35. Diagana, T: Existence of almost automorphic solutions to some neutral functional differential equations with infinite delay. Electron. J. Differ. Equ. 2008, 129 (2008)

36. N'Guérékata, G: Stepanov-like almost automorphic functions and monotone evolution equations. Nonlinear Anal. 68 2658-2667 (2008)

37. Ezzinbi, K, N'Guérékata, G: Massera type theorem for almost automorphic solutions of functional differential equations of neutral type. J. Math. Anal. Appl. 316, 707-721 (2006)

38. Hino, Y, Murakami, S: Almost automorphic solutions for abstract functional differential equations. J. Math. Anal. Appl. $286,741-752(2003)$

39. Diagana, T, Hernández, E, dos Santos, J: Existence of asymptotic almost automorphic solutions to some abstract partial neutral integro-differential equations. Nonlinear Anal., Theory Methods Appl. 71, 248-257 (2009) 
40. Shen, $W, Y i, Y$ : Almost automorphic and almost periodic dynamics in skew-product semiflows. Mem. Am. Math. Soc $136,647(1998)$

41. Abbas, S: Pseudo almost automorphic solutions of fractional order neutral differential equation. Semigroup Forum 81, 393-404 (2010)

42. Ezzinbi, K, N'Guérékata, G: Almost automorphic solutions for partial functional differential equations with infinite delay. Semigroup Forum 75, 95-115 (2007)

43. Chang, Y, Zhao, Z, Nieto, J: Pseudo almost automorphic and weighted pseudo almost automorphic mild solutions to semilinear differential equations in Hilbert spaces. Rev. Mat. Complut. 24, 421-438 (2011)

44. Mophou, G, N'Guérékata, G: On some classes of almost automorphic functions and applications to fractional differential equations. Comput. Math. Appl. 59, 1310-1317 (2010)

45. Ding, $\mathrm{H}$, Liang, J, Xiao, T: Some properties of Stepanov-like almost automorphic functions and applications to abstract evolution equations. Appl. Anal. 88, 1079-1091 (2009)

46. Chen, A, Chen, F, Deng, S: On almost automorphic mild solutions for fractional semilinear initial value problems. Comput. Math. Appl. 59, 1318-1325 (2010)

47. N'Guérékata, G: Almost Automorphic Functions and Almost Periodic Functions in Abstract Spaces. Kluwer Academic, New York (2001)

48. N'Guérékata, G: Topics in Almost Automorphy. Springer, New York (2005)

49. Liang, J, Zhang, J, Xiao, T: Composition of pseudo-almost automorphic and asymptotically almost automorphic functions. J. Math. Anal. Appl. 340, 1493-1499 (2008)

50. Liang, J, N'Guérékata, G, Xiao, T, Zhang, J: Some properties of pseudo-almost automorphic functions and applications to abstract differential equations. Nonlinear Anal., Theory Methods Appl. 70, 2731-2735 (2009)

51. Boulite, S, Maniar, L, N'Guérékata, G: Almost automorphic solutions for hyperbolic semilinear evolution equations. Semigroup Forum 71, 231-240 (2005)

52. Huang, Z, Gong, S, Wang, L: Positive almost periodic solution for a class of Lasota-Wazewska model with multiple time-varying delays. Comput. Math. Appl. 61, 755-760 (2011)

53. Cao, J, Yang, Q, Huang, Z: Optimal mild solutions and weighted pseudo-almost periodic classical solutions of fractional integro-differential equations. Nonlinear Anal., Theory Methods Appl. 74, 224-234 (2011)

54. Kilbas, A, Srivastava, H, Trujillo, J: Theory and Applications of Fractional Differential Equations. North-Holland Mathematics Studies, vol. 204. Elsevier, Amsterdam (2006)

55. Samko, S, Kilbas, A, Marichev, O: Fractional Integrals and Derivatives: Theory and Applications. Gordon \& Breach, New York (1993)

56. Diethelm, K: The Analysis of Fractional Differential Equations. Lecture Notes in Mathematics, vol. 2004. Springer, Berlin (2010)

57. Hilfer, R: Applications of Fractional Calculus in Physics. World Scientific, Singapore (2000)

58. Podlubny, I: Fractional Differential Equations. Academic Press, San Diego (1999)

59. Agarwal, R, Belmekki, M, Benchohra, M: A survey on semilinear differential equations and inclusions involving Riemann-Liouville fractional derivative. Adv. Differ. Equ. 2009, Article ID 981728 (2009). doi:10.1155/2009/981728

60. Agarwal, $R$, Lakshmikantham, $V$, Nieto, J: On the concept of solution for fractional differential equations with uncertainty. Nonlinear Anal., Theory Methods Appl. 72, 2859-2862 (2010)

61. Benchohra, M, Henderson, J, Ntouyas, S, Ouahab, A: Existence results for fractional order functional differential equations with infinite delay. J. Math. Anal. Appl. 338, 1340-1350 (2008)

62. Agarwal, R, Benchohra, M, Hamani, S: A survey on existence results for boundary value problems of nonlinear fractional differential equations and inclusions. Acta Appl. Math. 109, 973-1033 (2010)

63. El-Borai, M: Some probability densities and fundamental solutions of fractional evolution equations. Chaos Solitons Fractals 14, 433-440 (2002)

64. Lakshmikantham, V: Theory of fractional differential equations. Nonlinear Anal., Theory Methods Appl. 60, 3337-3343 (2008)

65. Lakshmikantham, V, Vatsala, A: Basic theory of fractional differential equations. Nonlinear Anal., Theory Methods Appl. 69, 2677-2682 (2008)

66. Lakshmikantham, V, Vatsala, A: Theory of fractional differential inequalities and applications. Commun. Appl. Anal. 11 395-402 (2007)

67. Lakshmikantham, V, Devi, J: Theory of fractional differential equations in Banach spaces. Eur. J. Pure Appl. Math. 1, 38-45 (2008)

68. Mophou, G, Nakoulima, O, N'Guérékata, G: Existence results for some fractional differential equations with nonlocal conditions. Nonlinear Stud. 17, 15-22 (2010)

69. Mophou, G, N'Guérékata, G: Existence of mild solution for some fractional differential equations with nonlocal conditions. Semigroup Forum 79, 315-322 (2009)

70. Mophou, G, N'Guérékata, G: On solutions of some nonlocal fractional differential equations with nondense domain. Nonlinear Anal., Theory Methods Appl. 71, 4668-4675 (2009)

71. Mophou, G: Existence and uniqueness of mild solutions to impulsive fractional differential equations. Nonlinear Anal., Theory Methods Appl. 72, 1604-1615 (2010)

72. N'Guérékata, G: A Cauchy problem for some fractional abstract differential equation with non local conditions. Nonlinear Anal., Theory Methods Appl. 70, 1873-1876 (2009)

73. Bazhlekova, E: Fractional Evolution Equations in Banach Spaces. Ph.D. thesis, Eindhoven University of Technology (2001)

74. Eidelman, S, Kochubei, A: Cauchy problem for fractional diffusion equations. J. Differ. Equ. 199, 211-255 (2004)

75. Gorenflo, R, Mainardi, F: Fractional calculus: integral and differential equations of fractional order. In: Carpinteri, A Mainardi, F (eds.) Fractals and Fractional Calculus in Continuum Mechanics. Springer, New York (1997)

76. Ahn, V, McVinisch, R: Fractional differential equations driven by Lévy noise. J. Appl. Math. Stoch. Anal. 16, 97-119 (2003)

77. Goldstein, J, N'Guérékata, G: Almost automorphic solutions of semilinear evolution equations. Proc. Am. Math. Soc. 133, 2401-2408 (2005) 
78. N'Guérékata, G: Existence and uniqueness of almost automorphic mild solutions of some semilinear abstract differential equations. Semigroup Forum 69, 80-86 (2004)

79. Cuevas, C, Lizama, C: Almost automorphic solutions to a class of semilinear fractional differential equations. Appl. Math. Lett. 21, 1315-1319 (2008)

80. Agarwal, R, Andrade, B, Cuevas, C: On type of periodicity and ergodicity to a class of fractional order differential equations. Adv. Differ. Equ. 2010, Article ID 179750 (2010). doi:10.1155/2010/179750

81. Agarwal, R, Cuevas, C, Soto, H: Pseudo-almost periodic solutions of a class of semilinear fractional differential equations. J. Appl. Math. Comput. 37, 625-634 (2011)

82. Cuevas, C, Souza, J: S-Asymptotically $\omega$-periodic solutions of semilinear fractional integro-differential equations. Appl. Math. Lett. 22, 865-870 (2009)

83. Cuevas, C, Souza, J: Existence of S-asymptotically $\omega$-periodic solutions for fractional order functional integro-differential equations with infinite delay. Nonlinear Anal., Theory Methods Appl. 72, 1683-1689 (2010)

84. Agarwal, R, de Andrade, B, Cuevas, C: Weighted pseudo-almost periodic solutions of a class of semilinear fractional differential equations. Nonlinear Anal., Real World Appl. 11, 3532-3554 (2010)

85. Cao, J, Yang, Q, Huang, Z: Existence of anti-periodic mild solutions for a class of semilinear fractional differential equations. Commun. Nonlinear Sci. Numer. Simul. 17, 277-283 (2012)

86. N'Guérékata, G: Comments on almost automorphic and almost periodic functions in Banach spaces. Far East J. Math. Sci.: FJMS 17, 337-344 (2005)

87. N'Guérékata, G, Pankov, A: Stepanov-like almost automorphic functions and monotone evolution equations. Nonlinear Anal., Theory Methods Appl. 68, 2658-2667 (2008)

88. Haase, M: The Functional Calculus for Sectorial Operators. Operator Theory: Advances and Applications, vol. 169. Birkhäuser, Basel (2006)

89. Cuesta, E: Asymptotic behaviour of the solutions of fractional integro-differential equations and some time discretizations. Discrete Contin. Dyn. Syst. suppl., 277-285 (2007)

90. Bugajewski, D, Diagana, T: Almost automorphy of the convolution operator and applications to differential and functional differential equations. Nonlinear Stud. 13, 129-140 (2006)

91. Cuevas, C, Pinto, M: Existence and uniqueness of pseudo almost periodic solutions of semilinear Cauchy problems with non dense domain. Nonlinear Anal., Theory Methods Appl. 45, 73-83 (2001)

\section{Submit your manuscript to a SpringerOpen ${ }^{\ominus}$ journal and benefit from:}

- Convenient online submission

- Rigorous peer review

- Immediate publication on acceptance

- Open access: articles freely available online

- High visibility within the field

Retaining the copyright to your article 